\title{
ANTIPODAL SETS IN ORIENTED REAL GRASSMANN MANIFOLDS
}

\author{
HIROYUKI TASAKI
}

\begin{abstract}
We reduce the problem of classifying all maximal antipodal sets in the oriented real Grassmann manifold $\tilde{G}_{k}\left(\mathbb{R}^{n}\right)$ to that of classifying all maximal subsets satisfying certain conditions in the set consisting of subsets of cardinality $k$ in $\{1, \ldots, n\}$. Using this reduction we classify all maximal antipodal sets in $\tilde{G}_{k}\left(\mathbb{R}^{n}\right)$ for $k \leq 4$. We construct some maximal antipodal subsets for higher $k$.
\end{abstract}

\section{INTRODUCTION}

Chen and Nagano [1] introduced the notion of antipodal sets in Riemannian symmetric spaces and showed some fundamental properties of antipodal sets. They also explicitly described antipodal sets in many compact Riemannian symmetric spaces, but they did not mention antipodal sets in oriented real Grassmann manifolds. In this paper we describe antipodal sets in oriented real Grassmann manifolds.

Let $M$ be a Riemannian symmetric space and denote by $s_{x}$ the geodesic symmetry of $M$ at $x \in M$. A subset $S$ in $M$ is called an antipodal set if $s_{x}(y)=y$ for any $x, y \in S$. We define the 2-number $\#_{2} M$ of $M$ as the supremum of $\# S$ for all antipodal sets $S$ in $M$. It is known that $\#_{2} M$ is finite. See Proposition 2.1 in Tanaka-Tasaki [4]. We call an antipodal set $S$ in $M$ great if $\#_{2} M=\# S$. A great antipodal set is maximal in all antipodal sets with respect to inclusion relation. In [4] we showed that any antipodal set in a symmetric $R$-space is included in a great antipodal set and any two great antipodal sets are congruent, however, antipodal sets in the oriented real Grassmann manifolds do not have such a nice property.

We denote by $\tilde{G}_{k}\left(\mathbb{R}^{n}\right)$ the oriented real Grassmann manifold consisting of oriented real vector subspaces of dimension $k$ in $\mathbb{R}^{n}$ equipped with the standard inner product. We reduce the problem of classifying all maximal antipodal sets in $\tilde{G}_{k}\left(\mathbb{R}^{n}\right)$ to that of classifying maximal

2010 Mathematics Subject Classification. 53C40 (Primary), 53D12 (Secondary).

Key words and phrases. antipodal set, oriented real Grassmann manifold, 2number, Riemannian symmetric space. 
subsets satisfying certain conditions in the set consisting of subsets of cardinality $k$ in $\{1, \ldots, n\}$. In the case where $k=1,2$ antipodal sets in $\tilde{G}_{k}\left(\mathbb{R}^{n}\right)$ are simple. In comparison with these we can see that antipodal sets in $\tilde{G}_{k}\left(\mathbb{R}^{n}\right)$ are not simple in the cases where $k \geq 3$ by using the reduction. In Sections 4,5 , and 6 we classify all of maximal antipodal sets of $\tilde{G}_{k}\left(\mathbb{R}^{n}\right)$ in the case where $k \leq 4$. In Section 7 we construct some maximal antipodal subsets for higher $k$.

\section{Real Grassmann manifolds}

The orthogonal group $O(n)$ acts transitively on $G_{k}\left(\mathbb{R}^{n}\right)$. We define an inner product on the Lie algebra $\mathfrak{o}(n)$ of $O(n)$ by

$$
\langle X, Y\rangle=\frac{1}{2} \operatorname{tr}\left({ }^{t} X Y\right) \quad(X, Y \in \mathfrak{o}(n))
$$

which induces a biinvariant Riemannian metric on $O(n)$ and an $O(n)$ invariant Riemannian metric on $G_{k}\left(\mathbb{R}^{n}\right)$. With respect to this Riemannian metric $G_{k}\left(\mathbb{R}^{n}\right)$ is a Riemannian symmetric space. It is known that $G_{k}\left(\mathbb{R}^{n}\right)$ is a symmetric $R$-space. For $V \in G_{k}\left(\mathbb{R}^{n}\right)$ the reflection $r_{V}$ with respect to $V$ is equal to $1_{V}-1_{V^{\perp}}$, which induces the geodesic symmetry $s_{V}$ of $G_{k}\left(\mathbb{R}^{n}\right)$ at $V$. We denote by $\left\langle v_{1}, \ldots, v_{m}\right\rangle$ the vector subspace of $\mathbb{R}^{n}$ spanned by $v_{1}, \ldots, v_{m} \in \mathbb{R}^{n}$. We denote by $\operatorname{Inc}_{k}(n)$ the set of all strictly increasing maps from $\{1, \ldots, k\}$ to $\{1, \ldots, n\}$. For a connected Riemannian manifold $M$, two subsets $X$ and $Y$ in $M$ are said to be congruent, if $X$ is transformed to $Y$ by an element of the identity component of the group of all isometries of $M$.

Lemma 2.1. Let $e_{1}, \ldots, e_{n}$ be an orthonormal basis of $\mathbb{R}^{n}$. The subset

$$
A=\left\{\left\langle e_{\alpha(1)}, \ldots, e_{\alpha(k)}\right\rangle \mid \alpha \in \operatorname{Inc}_{k}(n)\right\}
$$

in $G_{k}\left(\mathbb{R}^{n}\right)$ is a maximal antipodal set and any maximal antipodal set in $G_{k}\left(\mathbb{R}^{n}\right)$ is congruent with $A$. Thus $A$ is a great antipodal set and we have

$$
\#_{2} G_{k}\left(\mathbb{R}^{n}\right)=\# \operatorname{Inc}_{k}(n)=\left(\begin{array}{l}
n \\
k
\end{array}\right)
$$

This is showed in [1] and [4].

\section{ORIEnted REAl GRASSMAnN MANifolds}

We denote by $\tilde{G}_{k}\left(\mathbb{R}^{n}\right)$ the oriented real Grassmann manifold consisting of oriented vector subspaces of dimension $k$ in $\mathbb{R}^{n}$. In this case $O(n)$ also acts transitively on $\tilde{G}_{k}\left(\mathbb{R}^{n}\right)$. The biinvariant Riemannian metric on $O(n)$ defined in the previous section induces an $O(n)$-invariant Riemannian metric on $\tilde{G}_{k}\left(\mathbb{R}^{n}\right)$. With respect to this Riemannian metric 
$\tilde{G}_{k}\left(\mathbb{R}^{n}\right)$ is a Riemannian symmetric space, but it is not true in general that $\tilde{G}_{k}\left(\mathbb{R}^{n}\right)$ is a symmetric $R$-space.

We denote by $\bigwedge^{k} \mathbb{R}^{n}$ the exterior algebra of $\mathbb{R}^{n}$ of degree $k$. The inner product on $\mathbb{R}^{n}$ naturally induces an inner product on $\bigwedge^{k} \mathbb{R}^{n}$. With respect to this inner product

$$
\left\{e_{\alpha(1)} \wedge \cdots \wedge e_{\alpha(k)} \mid \alpha \in \operatorname{Inc}_{k}(n)\right\}
$$

is an orthonormal basis of $\bigwedge^{k} \mathbb{R}^{n}$, if $e_{1}, \ldots, e_{n}$ is an orthonormal basis of $\mathbb{R}^{n}$. We can regard $\tilde{G}_{k}\left(\mathbb{R}^{n}\right)$ as a Riemannian submanifold of $\bigwedge^{k} \mathbb{R}^{n}$ by identifying $\tilde{V} \in \tilde{G}_{k}\left(\mathbb{R}^{n}\right)$ with $v_{1} \wedge \cdots \wedge v_{k}$, where $v_{1}, \ldots, v_{k}$ is a positively oriented orthonormal basis of $\tilde{V}$.

For $\tilde{V} \in \tilde{G}_{k}\left(\mathbb{R}^{n}\right)$ we denote by $V$ the vector subspace determined by $\tilde{V}$. The reflection $r_{V}=1_{V}-1_{V^{\perp}}$ induces the geodesic symmetry $s_{\tilde{V}}$ of $\tilde{G}_{k}\left(\mathbb{R}^{n}\right)$ at $\tilde{V}$ defined by

$$
s_{\tilde{V}}\left(v_{1} \wedge \cdots \wedge v_{k}\right)=r_{V}\left(v_{1}\right) \wedge \cdots \wedge r_{V}\left(v_{k}\right) .
$$

This is equal to $\left(\wedge^{k} r_{V}\right)\left(v_{1} \wedge \cdots \wedge v_{k}\right)$. Hence the geodesic symmetry $s_{\tilde{V}}$ is the restriction of the linear transformation $\wedge^{k} r_{V}$ of $\bigwedge^{k} \mathbb{R}^{n}$ to $\tilde{G}_{k}\left(\mathbb{R}^{n}\right)$. We define a double covering map $p: \tilde{G}_{k}\left(\mathbb{R}^{n}\right) \rightarrow G_{k}\left(\mathbb{R}^{n}\right)$ by $p(\tilde{V})=V$. We have $s_{p(x)} \circ p=p \circ s_{x}$ for $x \in \tilde{G}_{k}\left(\mathbb{R}^{n}\right)$, because $p$ is an isometric covering map.

Lemma 3.1. For any antipodal set $S$ in $\tilde{G}_{k}\left(\mathbb{R}^{n}\right)$ there exists an orthonormal basis $v_{1}, \ldots, v_{n}$ of $\mathbb{R}^{n}$ which satisfies

$$
S \subset\left\{ \pm v_{\alpha(1)} \wedge \cdots \wedge v_{\alpha(k)} \mid \alpha \in \operatorname{Inc}_{k}(n)\right\},
$$

where we regard $\tilde{G}_{k}\left(\mathbb{R}^{n}\right)$ as a submanifold of $\bigwedge^{k} \mathbb{R}^{n}$.

Proof. For any $x, y \in S$ we have

$$
s_{p(x)}(p(y))=p\left(s_{x}(y)\right)=p(y),
$$

thus $p(S)$ is an antipodal set in $G_{k}\left(\mathbb{R}^{n}\right)$. According to Lemma 2.1 there exists an orthonormal basis $v_{1}, \ldots, v_{n}$ of $\mathbb{R}^{n}$ which satisfies

$$
p(S) \subset\left\{\left\langle v_{\alpha(1)}, \ldots, v_{\alpha(k)}\right\rangle \mid \alpha \in \operatorname{Inc}_{k}(n)\right\} .
$$

Hence we get

$$
S \subset\left\{ \pm v_{\alpha(1)} \wedge \cdots \wedge v_{\alpha(k)} \mid \alpha \in \operatorname{Inc}_{k}(n)\right\} .
$$


For a set $X$ we denote by $P_{k}(X)$ the set of all subsets $\alpha$ in $X$ whose cardinalities $\# \alpha$ are equal to $k$. We simply denote $P_{k}(n)=$ $P_{k}(\{1, \ldots, n\})$. The map

$$
\operatorname{Inc}_{k}(n) \rightarrow P_{k}(n) ; \alpha \mapsto\{\alpha(1), \ldots, \alpha(k)\}
$$

is bijective and we identify $\operatorname{Inc}_{k}(n)$ with $P_{k}(n)$ by this bijective map.

Lemma 3.2. For any maximal antipodal set $S$ in $\tilde{G}_{k}\left(\mathbb{R}^{n}\right)$ there exist an orthonormal basis $v_{1}, \ldots, v_{n}$ of $\mathbb{R}^{n}$ and a subset $A$ of $P_{k}(n)$ which satisfy

$$
S=\left\{ \pm v_{\alpha(1)} \wedge \cdots \wedge v_{\alpha(k)} \mid \alpha \in A\right\} .
$$

Proof. By Lemma 3.1, there exists an orthonormal basis $v_{1}, \ldots, v_{n}$ of $\mathbb{R}^{n}$ which satisfies

$$
S \subset\left\{ \pm v_{\alpha(1)} \wedge \cdots \wedge v_{\alpha(k)} \mid \alpha \in \operatorname{Inc}_{k}(n)\right\} .
$$

We set $\vec{v}_{\alpha}=v_{\alpha(1)} \wedge \cdots \wedge v_{\alpha(k)}$ and

$$
A=\left\{\alpha \in P_{k}(n) \mid \vec{v}_{\alpha} \in S \text { or }-\vec{v}_{\alpha} \in S\right\} .
$$

This definition of $A$ implies $S \subset\left\{ \pm \vec{v}_{\alpha} \mid \alpha \in A\right\}$.

If $x, y \in S$ then $s_{x}(y)=y$. In this case we have $s_{x}(-y)=-s_{x}(y)=$ $-y$, because $s_{x}$ is the restriction of a linear transformation of $\bigwedge^{k} \mathbb{R}^{n}$. By the definition we have $s_{-y}=s_{y}$ and $S \cup\{-y\}$ is also an antipodal set. Since $S$ is a maximal antipodal set, we have $S=\left\{ \pm \vec{v}_{\alpha} \mid \alpha \in A\right\}$.

For an orthonormal basis $\boldsymbol{v}=\left\{v_{1}, \ldots, v_{n}\right\}$ of $\mathbb{R}^{n}$ and a subset $A$ of $P_{k}(n)$ we define

$$
\mathcal{A}_{\boldsymbol{v}}(A)=\left\{ \pm \vec{v}_{\alpha} \mid \alpha \in A\right\} .
$$

We have to determine which subset $A$ of $P_{k}(n)$ defines a maximal antipodal set $\mathcal{A}_{\boldsymbol{v}}(A)$ in $\tilde{G}_{k}\left(\mathbb{R}^{n}\right)$.

Lemma 3.3. Let $\boldsymbol{v}=\left\{v_{1}, \ldots, v_{n}\right\}$ be an orthonormal basis of $\mathbb{R}^{n}$ and $A$ be a subset of $P_{k}(n) . \mathcal{A}_{\boldsymbol{v}}(A)$ is an antipodal set in $\tilde{G}_{k}\left(\mathbb{R}^{n}\right)$ if and only if the cardinality $\#(\beta-\alpha)$ is even for any $\alpha, \beta \in A$, where $\beta-\alpha=$ $\{b \in \beta \mid b \notin \alpha\}$.

Proof. For $\alpha, \beta \in P_{k}(n)$ we have

$$
s_{\vec{v}_{\alpha}}\left(\vec{v}_{\beta}\right)=r_{p\left(\vec{v}_{\alpha}\right)} v_{\beta(1)} \wedge \cdots \wedge r_{p\left(\vec{v}_{\alpha}\right)} v_{\beta(k)}=(-1)^{\#(\beta-\alpha)} \vec{v}_{\beta} .
$$

So $s_{\vec{v}_{\alpha}}\left(\vec{v}_{\beta}\right)=\vec{v}_{\beta}$ if and only if $\#(\beta-\alpha)$ is even. Hence $\mathcal{A}_{\boldsymbol{v}}(A)$ is an antipodal set in $\tilde{G}_{k}\left(\mathbb{R}^{n}\right)$ if and only if the cardinality $\#(\beta-\alpha)$ is even for any $\alpha, \beta \in A$.

By the above lemma we introduce the following definition. 
Definition 3.1. $\alpha, \beta \in P_{k}(n)$ are antipodal, if the cardinality $\#(\beta-\alpha)$ is even. A subset $A$ of $P_{k}(n)$ is said to be antipodal, if any $\alpha, \beta \in A$ are antipodal. By the definition $\alpha, \beta \in P_{k}(n)$ are antipodal if and only if $\#(\alpha \cap \beta) \equiv k(\bmod 2)$

We denote by $\operatorname{Sym}(X)$ the symmetric group on a finite set $X$. If $X=\{1, \ldots, n\}$, we simply write $\operatorname{Sym}(n)=\operatorname{Sym}(X)$ Two subsets $X$ and $Y$ in $P_{k}(X)$ are said to be congruent, if $X$ is transformed to $Y$ by an element of $\operatorname{Sym}(X)$.

If $A \subset B \subset P_{k}(n)$, then $\mathcal{A}_{\boldsymbol{v}}(A) \subset \mathcal{A}_{\boldsymbol{v}}(B)$. This implication and the lemmas mentioned above imply the following theorem.

Theorem 3.1. If $v_{1}, \ldots, v_{n}$ is an orthonormal basis of $\mathbb{R}^{n}$ and $A$ is a maximal antipodal subset of $P_{k}(n)$, then $\mathcal{A}_{\boldsymbol{v}}(A)$ is a maximal antipodal set in $\tilde{G}_{k}\left(\mathbb{R}^{n}\right)$. Conversely for any maximal antipodal set $S$ in $\tilde{G}_{k}\left(\mathbb{R}^{n}\right)$ there exist an orthonormal basis $v_{1}, \ldots, v_{n}$ of $\mathbb{R}^{n}$ and a maximal antipodal subset $A$ of $P_{k}(n)$ which satisfy $S=\mathcal{A}_{\boldsymbol{v}}(A)$. Moreover the correspondence above induces a bijection from the set of all congruent classes of maximal antipodal subsets in $P_{k}(n)$ to the set of all congruent classes of maximal antipodal sets in $\tilde{G}_{k}\left(\mathbb{R}^{n}\right)$.

\section{Corollary 3.1.}

$\#{ }_{2} \tilde{G}_{k}\left(\mathbb{R}^{n}\right)=2 \max \left\{\# A \mid A\right.$ is a maximal antipodal subset of $\left.P_{k}(n)\right\}$,

In order to determine maximal antipodal sets in $\tilde{G}_{k}\left(\mathbb{R}^{n}\right)$ we have to determine maximal antipodal subsets of $P_{k}(n)$. We investigate maximal antipodal subsets of $P_{k}(n)$ in the sequel sections.

\section{Maximal Antipodal SUbSets of $P_{k}(n)$}

In this section we consider a strategy to determine all congruent classes of maximal antipodal subsets in $P_{k}(n)$.

We denote by $\alpha^{c}$ the complement of $\alpha \in P_{k}(n)$ in $\{1, \ldots, n\}$. For $\alpha, \beta \in P_{k}(n)$ we have $\beta-\alpha=\alpha^{c}-\beta^{c}$, which implies the following lemma.

Lemma 4.1. A subset $A \subset P_{k}(n)$ is antipodal if and only if $A^{c}=$ $\left\{\alpha^{c} \mid \alpha \in A\right\} \subset P_{n-k}(n)$ is antipodal. Moreover $A$ is a maximal antipodal subset of $P_{k}(n)$ if and only if $A^{c}$ is a maximal antipodal subset of $P_{n-k}(n)$.

We identify $P_{k}(n)$ with $\operatorname{Inc}_{k}(n)$ and define the lexicographic order on $P_{k}(n)$. With respect to this order $\{1,2, \ldots, k\}$ is the minimum element. From now on we describe how to construct representatives of all congruent classes of maximal antipodal subsets of $P_{k}(n)$. At first we 
take $A_{1}=\{\{1,2, \ldots, k\}\}$. Next we construct some antipodal subsets of cardinality $i+1$ from an antipodal subset $A_{i}$ of cardinality $i$. We define the stabilizer $S\left(A_{i}\right)$ of $A_{i}$ by

$$
S\left(A_{i}\right)=\left\{g \in \operatorname{Sym}(n) \mid g\left(A_{i}\right)=A_{i}\right\} .
$$

We consider the set of elements which are antipodal to $A_{i}$ :

$$
A\left(A_{i}\right)=\left\{\alpha \in P_{k}(n)-A_{i} \mid A_{i} \cup\{\alpha\} \text { is antipodal }\right\} .
$$

If $A\left(A_{i}\right)$ is empty, $A_{i}$ is a maximal antipodal subset of $P_{k}(n)$ and the procedure in the case of the orbit $A_{i}$ ends. So we consider the case where $A\left(A_{i}\right)$ is not empty. $S\left(A_{i}\right)$ stabilizes $A_{i}$ and preserves the antipodal relations, hence $S\left(A_{i}\right)$ stabilizes $A\left(A_{i}\right)$, too. We decompose $A\left(A_{i}\right)$ to a disjoint union of the orbits $O_{1}, \ldots, O_{j}$ of $S\left(A_{i}\right)$. We take the minimum element $\alpha_{a}$ of $O_{a}$ for each $1 \leq a \leq j$, add $\alpha_{a}$ to $A_{i}$ and obtain $A_{i+1, a}=A_{i} \cup\left\{\alpha_{a}\right\}$. Since $\alpha_{a}$ belongs to $A\left(A_{i}\right)$, each $A_{i+1, a}$ is antipodal. We divide the procedure to the cases by the orbits of $S\left(A_{i}\right)$. We take each $A_{i+1, a}$ as $A_{i+1}$ and repeat the above procedure until reaching a maximal antipodal subset of $P_{k}(n)$.

Lemma 4.2. We can obtain representatives of all congruent classes of maximal antipodal subsets of $P_{k}(n)$ by the procedure mentioned above.

Proof. Let $A$ be a maximal antipodal subset of $P_{k}(n)$. Transforming $A$ by an element of $\operatorname{Sym}(n)$ we can suppose that $A_{1} \subset A$. Transforming $A$ by an element of $S\left(A_{1}\right)$ we can take the minimum element $\alpha$ of an orbit of $S\left(A_{1}\right)$ in $A\left(A_{1}\right)$ which belongs to $A$. The second antipodal subset $A_{2}$ satisfies $A_{2}=A_{1} \cup\{\alpha\} \subset A$. We can repeat this procedure until $A_{i}=A$ for $i=\# A$.

Remark 4.1. If there exists a subset $B$ of $A\left(A_{j}\right)$ satisfying that any element of $B$ and any other element of $A\left(A_{j}\right)$ are antipodal in the procedure mentioned above, then we can add $B$ to $A_{j}$ and obtain $A_{j+\# B}=A_{j} \cup B$.

It may happen that two of the maximal antipodal subsets obtained by the procedure mentioned above are congruent. It actually happens in the proof of Theorem 6.1, which classify maximal antipodal subsets of $P_{4}(n)$.

In the case where $k=1$ any two different elements in $P_{1}(n)$ are not antipodal, so we get the following proposition.

Proposition 4.1. $\{\{1\}\}$ is a maximal antipodal subset of $P_{1}(n)$. Conversely any maximal antipodal subset of $P_{1}(n)$ is congruent with it. 
The corresponding maximal antipodal set of $\tilde{G}_{1}\left(\mathbb{R}^{n}\right)=S^{n-1}$ is $\{ \pm v\}$ for $v \in S^{n-1}$. This proposition and Corollary 3.1 imply the following corollary.

Corollary 4.1. $\#_{2} S^{n-1}=2$.

Proposition 4.2. For a natural number $l$ we put

$$
A(2,2 l)=\{\{1,2\},\{3,4\}, \ldots,\{2 l-1,2 l\}\} .
$$

$A(2,2[n / 2])$ is a maximal antipodal subset of $P_{2}(n)$. Conversely any maximal antipodal subset of $P_{2}(n)$ is congruent with it.

Proof. Two different elements $\alpha, \beta$ in $P_{2}(n)$ are antipodal if and only if $\alpha \cap \beta=\emptyset$. We take $A_{1}=\{\{1,2\}\}$. We have $A\left(A_{1}\right)=P_{2}(\{3, \ldots, n\})$ and it is an orbit of $S\left(A_{1}\right)$. We take the minimum element $\{3,4\}$ of $A\left(A_{1}\right)$, add it to $A_{1}$ and obtain $A_{2}=\{\{1,2\},\{3,4\}\}$. We can repeat this procedure until we get

$$
\{\{1,2\},\{3,4\}, \ldots,\{2[n / 2]-1,2[n / 2]\}\} .
$$

Hence it is a maximal antipodal subset of $P_{2}(n)$, and any maximal antipodal subset of $P_{2}(n)$ is congruent with it.

Corollary 4.2. \# $\tilde{G}_{2}\left(\mathbb{R}^{n}\right)=2[n / 2]$.

Remark 4.2. We can construct a 2 -form from a subset $A$ of $P_{2}(2 l)$ and an orthonormal basis $\left\{e_{i}\right\}$ of $\mathbb{R}^{2 l}$ as follows:

$$
\sum_{\alpha \in A} \vec{e}_{\alpha}^{*}=\sum_{\alpha \in A} e_{\alpha(1)}^{*} \wedge e_{\alpha(2)}^{*}
$$

The 2-form constructed from the maximal antipodal subset $\{\{1,2\}, \ldots,\{2 l-$ $1,2 l\}\}$ of $P_{2}(2 l)$ is the Kähler form

$$
e_{1}^{*} \wedge e_{2}^{*}+\cdots+e_{2 l-1}^{*} \wedge e_{2 l}^{*}
$$

on $\mathbb{C}^{l}=\mathbb{R}^{2 l}$ with a suitable Hermitian structure.

In comparison with the cases where $k=1$ and $k=2$, the procedure of constructing maximal antipodal subsets of $P_{k}(n)$ is not simple in the cases where $k$ is greater than 2 . 


\section{Maximal antipodal subsets of $P_{3}(n)$}

In the case where $k=3$, two different elements $\alpha, \beta$ in $P_{3}(n)$ are antipodal if and only if $\#(\alpha \cap \beta)=1$. Let

$$
\begin{aligned}
A(3,2 l+1)= & \{\{1,2,3\},\{1,4,5\}, \ldots,\{1,2 l, 2 l+1\}\}, \\
B(3,6)= & \{\{1,2,3\},\{1,4,5\},\{2,4,6\},\{3,5,6\}\}, \\
B(3,7)= & \{\{1,2,3\},\{1,4,5\},\{2,4,6\},\{3,5,6\}, \\
& \{1,6,7\},\{2,5,7\},\{3,4,7\}\} .
\end{aligned}
$$

These are antipodal subsets in $P_{3}(2 l+1), P_{3}(6)$ and $P_{3}(7)$ respectively. These satisfy

$$
A(3,5) \subset B(3,6) \subset B(3,7), \quad A(3,5) \subset A(3,7) \subset B(3,7) .
$$

These antipodal subsets can be visualized by Figure 1 .
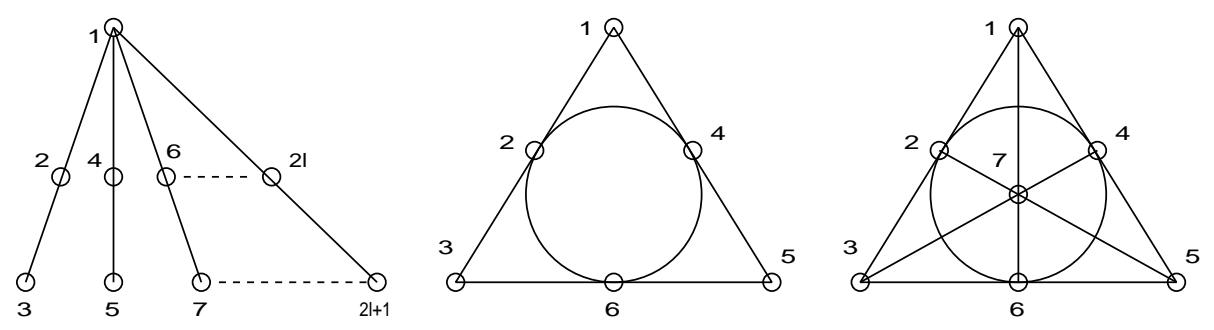

Figure 1. $A(3,2 l+1), B(3,6)$ and $B(3,7)$

As stated in Theorem 5.1, $A(3,2 l+1)$ is a maximal antipodal subset in $P_{3}(2 l+1)$ and $P_{3}(2 l+2)$ except for the cases of $A(3,5) \subset P_{3}(6)$ and $A(3,7) \subset P_{3}(7), P_{3}(8)$. The relation of inclusions mentioned above shows that these are not maximal.

Remark 5.1. The intersection relation between elements in $B(3,7)$ is equal to that between projective lines in the projective plane $F_{2} P^{2}$ over the binary field $F_{2}$ consisting of 0 and 1.

Theorem 5.1. Let $l=[(n-1) / 2]$. Each in the following table is a maximal antipodal subset of $P_{3}(n)$ :

\begin{tabular}{|c|c|c|c|c|c|}
\hline$n$ & 3,4 & 5 & 6 & 7,8 & more than 8 \\
\hline & $A(3,3)$ & $A(3,5)$ & $B(3,6)$ & $B(3,7)$ & $A(3,2 l+1), B(3,7)$ \\
\hline
\end{tabular}

Conversely any maximal antipodal subset of $P_{3}(n)$ is congruent with one of them.

Before proving the theorem we prepare some notation. For subsets $A, B \subset X$ satisfying $A \cap B=\emptyset$, we denote

$$
P_{k}(A) \times P_{l}(B)=\left\{\alpha \cup \beta \mid \alpha \in P_{k}(A), \beta \in P_{l}(B)\right\} \subset P_{k+l}(X) .
$$


For more than two subsets in $X$ we can similarly define $P_{k_{1}}\left(A_{1}\right) \times \cdots \times$ $P_{k_{l}}\left(A_{l}\right)$.

Proof. We take $A_{1}=\{\{1,2,3\}\}$. We have

$$
A\left(A_{1}\right)=P_{1}(\{1,2,3\}) \times P_{2}(\{4, \ldots, n\}) .
$$

If $n \leq 4, A\left(A_{1}\right)$ is empty and $A_{1}=A(3,3)$ is a maximal antipodal subset of $P_{3}(n)$. Thus we suppose that $n \geq 5$. The stabilizer $S\left(A_{1}\right)$ of $A_{1}$ is equal to $\operatorname{Sym}(\{1,2,3\}) \times \operatorname{Sym}(\{4, \ldots, n\})$. So $A\left(A_{1}\right)$ is an orbit of $S\left(A_{1}\right)$. We take the minimum element $\{1,4,5\}$ of $A\left(A_{1}\right)$, add it to $A_{1}$ and obtain $A_{2}=\{\{1,2,3\},\{1,4,5\}\}=A(3,5)$.

$$
\begin{aligned}
A\left(A_{2}\right)= & \left\{\alpha \in A\left(A_{1}\right)-\{\{1,4,5\}\} \mid \alpha \text { and }\{1,4,5\} \text { are antipodal }\right\} \\
= & P_{1}(\{2,3\}) \times P_{1}(\{4,5\}) \times P_{1}(\{6, \ldots, n\}) \\
& \cup P_{1}(\{1\}) \times P_{2}(\{6, \ldots, n\}) .
\end{aligned}
$$

If $n=5, A\left(A_{2}\right)$ is empty and $A_{2}=A(3,5)$ is a maximal antipodal subset of $P_{3}(5)$. Thus we suppose that $n \geq 6$. The stabilizer $S\left(A_{2}\right)$ fixes 1 and induces permutations on $\{\{2,3\},\{4,5\}\}$, so it acts transitively on $P_{1}(\{2,3\}) \times P_{1}(\{4,5\}) \times P_{1}(\{6, \ldots, n\})$ and $P_{1}(\{1\}) \times P_{2}(\{6, \ldots, n\})$. These are two orbits of $S\left(A_{2}\right)$. If $n=6$, then the second orbit is empty and $A\left(A_{2}\right)=P_{1}(\{2,3\}) \times P_{1}(\{4,5\}) \times P_{1}(\{6\})$. We take the minimum element $\{2,4,6\}$ of the orbit $A\left(A_{2}\right)$, add it to $A_{2}$ and obtain $A_{3,1}=\{\{1,2,3\},\{1,4,5\},\{2,4,6\}\}$.

$$
\begin{aligned}
A\left(A_{3,1}\right) & =\left\{\alpha \in A\left(A_{2}\right)-\{\{2,4,6\}\} \mid \alpha \text { and }\{2,4,6\} \text { are antipodal }\right\} \\
& =\{\{3,5,6\}\} .
\end{aligned}
$$

We add $\{3,5,6\}$ to $A_{3,1}$ and obtain

$$
\{\{1,2,3\},\{1,4,5\},\{2,4,6\},\{3,5,6\}\}=B(3,6),
$$

which is a maximal antipodal subset of $P_{3}(6)$. We have obtained representatives of all congruent classes of maximal antipodal subsets in $P_{3}(n)$ for $n \leq 6$.

We suppose that $n \geq 7$. In this case $A\left(A_{2}\right)$ has two orbits of $S\left(A_{2}\right)$ :

$$
\begin{aligned}
& P_{1}(\{2,3\}) \times P_{1}(\{4,5\}) \times P_{1}(\{6, \ldots, n\}), \\
& P_{1}(\{1\}) \times P_{2}(\{6, \ldots, n\}),
\end{aligned}
$$

which are not empty. Hence we divide the procedure to two cases where we take minimum elements in the two orbits.

(1) We take the minimum element $\{2,4,6\}$ of the orbit $P_{1}(\{2,3\}) \times$ $P_{1}(\{4,5\}) \times P_{1}(\{6, \ldots, n\})$, add it to $A_{2}$ and obtain

$$
A_{3,1}=\{\{1,2,3\},\{1,4,5\},\{2,4,6\}\} .
$$


We have

$$
\begin{aligned}
A\left(A_{3,1}\right) & \\
= & \left\{\alpha \in A\left(A_{2}\right)-\{\{2,4,6\}\} \mid \alpha \text { and }\{2,4,6\} \text { are antipodal }\right\} \\
= & \{\{3,5,6\}\} \cup P_{1}(\{2\}) \times P_{1}(\{5\}) \times P_{1}(\{7, \ldots, n\}) \\
& \cup P_{1}(\{3\}) \times P_{1}(\{4\}) \times P_{1}(\{7, \ldots, n\}) \\
& \cup P_{1}(\{1\}) \times P_{1}(\{6\}) \times P_{1}(\{7, \ldots, n\}) \\
= & \{\{3,5,6\}\} \cup\{\{1,6\},\{2,5\},\{3,4\}\} \times P_{1}(\{7, \ldots, n\}) .
\end{aligned}
$$

In $A\left(A_{3,1}\right),\{3,5,6\}$ and other elements are antipodal. We can add it to $A_{3,1}$ and obtain

$$
A_{4,1}=\{\{1,2,3\},\{1,4,5\},\{2,4,6\},\{3,5,6\}\}=B(3,6) .
$$

We have

$$
A\left(A_{4,1}\right)=\{\{1,6\},\{2,5\},\{3,4\}\} \times P_{1}(\{7, \ldots, n\}) .
$$

The stabilizer $S\left(A_{4,1}\right)$ induces permutations on

$$
\{\{1,6\},\{2,5\},\{3,4\}\},
$$

so it acts transitively on $A\left(A_{4,1}\right)$. This is an orbit of $S\left(A_{4,1}\right)$. We take the minimum element $\{1,6,7\}$ of $A\left(A_{4,1}\right)$, add it to $A_{4,1}$ and obtain

$$
A_{5,1}=\{\{1,2,3\},\{1,4,5\},\{2,4,6\},\{3,5,6\},\{1,6,7\}\} .
$$

We have

$$
A\left(A_{5,1}\right)=\{\{2,5,7\},\{3,4,7\}\} .
$$

These two elements are antipodal. We can add these to $A_{5,1}$ and obtain

$$
\begin{aligned}
& \{\{1,2,3\},\{1,4,5\},\{2,4,6\},\{3,5,6\},\{1,6,7\},\{2,5,7\},\{3,4,7\}\} \\
& =B(3,7),
\end{aligned}
$$

which is a maximal antipodal subset of $P_{3}(n)$ for $n \geq 7$.

(2) We take the minimum element $\{1,6,7\}$ of the orbit $P_{1}(\{1\}) \times$ $P_{2}(\{6, \ldots, n\})$, add it to $A_{2}$ and obtain

$$
A_{3,2}=\{\{1,2,3\},\{1,4,5\},\{1,6,7\}\} .
$$

We have

$$
\begin{aligned}
A\left(A_{3,2}\right)= & P_{1}(\{2,3\}) \times P_{1}(\{4,5\}) \times P_{1}(\{6,7\}) \\
& \cup P_{1}(\{1\}) \times P_{2}(\{8, \ldots, n\}) .
\end{aligned}
$$

The stabilizer $S\left(A_{3,2}\right)$ fixes 1 and induces permutations on

$$
\{\{2,3\},\{4,5\},\{6,7\}\} \text {, }
$$


so it acts transitively on $P_{1}(\{2,3\}) \times P_{1}(\{4,5\}) \times P_{1}(\{6,7\})$ and $P_{1}(\{1\}) \times$ $P_{2}(\{8, \ldots, n\})$. These are two orbits of $S\left(A_{3,2}\right)$. If $n \leq 8$, then the second orbit is empty and $A\left(A_{3,2}\right)=P_{1}(\{2,3\}) \times P_{1}(\{4,5\}) \times P_{1}(\{6,7\})$. We take the minimum element $\{2,4,6\}$, add it to $A_{3,2}$ and obtain

$$
A_{4,2}=\{\{1,2,3\},\{1,4,5\},\{1,6,7\},\{2,4,6\}\} .
$$

We have

$$
A\left(A_{4,2}\right)=\{\{2,5,7\},\{3,4,7\},\{3,5,6\}\} .
$$

These are antipodal to each other. We can add these to $A_{4,2}$ and obtain $B(3,7)$. As we have showed in the case $(1), B(3,7)$ is a maximal antipodal subset of $P_{3}(n)$ for $n \geq 7$. We have obtained representatives of all congruent classes of maximal antipodal subsets in $P_{3}(n)$ for $n \leq 8$.

We suppose that $n \geq 9$. In this case $A\left(A_{3,2}\right)$ has two orbits of $S\left(A_{3,2}\right)$ :

$$
\begin{aligned}
& P_{1}(\{2,3\}) \times P_{1}(\{4,5\}) \times P_{1}(\{6,7\}), \\
& P_{1}(\{1\}) \times P_{2}(\{8, \ldots, n\}),
\end{aligned}
$$

which are not empty. If we take the minimum element $\{2,4,6\}$, then we reach $B(3,7)$ in a way similar to the above argument. Hence we take the minimum element $\{1,8,9\}$ of the second orbit $P_{1}(\{1\}) \times P_{2}(\{8, \ldots, n\})$, add it to $A_{3,2}$ and obtain

$$
A_{4,3}=\{\{1,2,3\},\{1,4,5\},\{1,6,7\},\{1,8,9\}\} .
$$

We have

$$
A\left(A_{4,3}\right)=P_{1}(\{1\}) \times P_{2}(\{10, \ldots, n\}) .
$$

The stabilizer $S\left(A_{4,3}\right)$ fixes 1 and induces permutations on

$$
\{\{2,3\},\{4,5\},\{6,7\},\{8,9\}\},
$$

so it acts transitively on $A\left(A_{4,3}\right)$. We can repeat this procedure until we get $A(3,2 l+1)$, where $l=[(n-1) / 2]$. Therefore we complete the proof of the theorem.

Corollary 5.1. The 2-number $\#_{2} \tilde{G}_{3}\left(\mathbb{R}^{n}\right)$ is as follows:

\begin{tabular}{|c|c|c|c|c|c|}
\hline$n$ & 4 & 5 & 6 & $7, \ldots, 16$ & more than 16 \\
\hline$\#_{2} \tilde{G}_{3}\left(\mathbb{R}^{n}\right)$ & 2 & 4 & 8 & 14 & $2[(n-1) / 2]$ \\
\hline
\end{tabular}

Proof. We have

$$
\# A(3,2 l+1)=l, \quad \# B(3,6)=4, \quad \# B(3,7)=7
$$

and obtain the corollary by Corollary 3.1. 
Remark 5.2. We can construct 3-forms from maximal antipodal subsets of $P_{3}(n)$ in a way similar to Remark 4.2. The special Lagrangian 3 -form on $\mathbb{C}^{3}$ is defined by

$$
\begin{aligned}
& \operatorname{Re}\left[\left(e_{1}^{*}+\sqrt{-1} e_{2}^{*}\right) \wedge\left(e_{3}^{*}+\sqrt{-1} e_{4}^{*}\right) \wedge\left(e_{5}^{*}+\sqrt{-1} e_{6}^{*}\right)\right] \\
& =e_{1}^{*} \wedge e_{3}^{*} \wedge e_{5}^{*}-e_{1}^{*} \wedge e_{4}^{*} \wedge e_{6}^{*}-e_{2}^{*} \wedge e_{3}^{*} \wedge e_{6}^{*}-e_{2}^{*} \wedge e_{4}^{*} \wedge e_{5}^{*},
\end{aligned}
$$

which corresponds to $\{\{1,3,5\},\{1,4,6\},\{2,3,6\},\{2,4,5\}\}$. This is a maximal antipodal subset of $P_{3}(6)$, so it is congruent with $B(3,6)$ by Theorem 5.1. We can also directly see that the subset transformed by acting the permutation $\left(\begin{array}{llllll}1 & 2 & 3 & 4 & 5 & 6 \\ 1 & 6 & 2 & 5 & 3 & 4\end{array}\right)$ on it is equal to $B(3,6)$. The 3 -form constructed from $B(3,7)$ is equal to

$$
\begin{aligned}
& e_{1}^{*} \wedge e_{2}^{*} \wedge e_{3}^{*}+e_{1}^{*} \wedge e_{4}^{*} \wedge e_{5}^{*}+e_{2}^{*} \wedge e_{4}^{*} \wedge e_{6}^{*}+e_{3}^{*} \wedge e_{5}^{*} \wedge e_{6}^{*}+e_{1}^{*} \wedge e_{6}^{*} \wedge e_{7}^{*} \\
& +e_{2}^{*} \wedge e_{5}^{*} \wedge e_{7}^{*}+e_{3}^{*} \wedge e_{4}^{*} \wedge e_{7}^{*},
\end{aligned}
$$

which was found by Harvey-Lawson [2] as a 3-form on $\operatorname{Im} \mathbb{O}=\mathbb{R}^{7}$ invariant under the action of the exceptional compact simple Lie group $G_{2}$ on $\operatorname{ImO}$.

\section{Maximal Antipodal subsets of $P_{4}(n)$}

We define three antipodal subsets of $P_{4}(n)$ as follows:

$$
\begin{aligned}
A(4,2 l) & =\left\{\alpha \cup \beta \in P_{4}(2 l) \mid \alpha, \beta \in\{\{1,2\},\{3,4\}, \ldots,\{2 l-1,2 l\}\}\right\}, \\
B(4,7) & =B^{c}(3,7)=\left\{\alpha^{c} \mid \alpha \in B(3,7)\right\}, \\
B(4,8) & =B(4,7) \cup B(3,7) \times\{\{8\}\} .
\end{aligned}
$$

It is easy to see that $A(4,2 l)$ is antipodal. Lemma 4.1 and Theorem 5.1 imply that $B(4,7)$ is a maximal antipodal subset of $P_{4}(7)$. Since $B(3,7)$ is antipodal in $P_{3}(7)$, so is $B(3,7) \times\{\{8\}\}$ in $P_{4}(8)$. For $\alpha, \beta \in B(3,7)$ we have $\alpha^{c} \cap(\beta \cup\{8\})=\beta-\alpha$. Thus $B(4,8)$ is antipodal.

As stated in Theorem 6.1, $A(4,2 l)$ is a maximal antipodal subset in $P_{4}(2 l)$ and $P_{4}(2 l+1)$ except for the cases of $A(4,6) \subset P_{4}(7)$ and $A(4,8) \subset P_{4}(8), P_{4}(9)$. We can directly show that these are not maximal.

$$
B(4,7) \supset\left\{\alpha \cup \beta \in P_{4}(7) \mid \alpha, \beta \in\{\{2,3\},\{4,5\},\{6,7\}\}\right\},
$$

which is congruent with $A(4,6)$ in $P_{4}(7)$. Thus $A(4,6)$ is not a maximal antipodal subset in $P_{4}(7)$.

$$
B(4,8) \supset\left\{\alpha \cup \beta \in P_{4}(7) \mid \alpha, \beta \in\{\{2,3\},\{4,5\},\{6,7\},\{1,8\}\}\right\},
$$

which is congruent with $A(4,8)$ in $P_{4}(8)$. Thus $A(4,8)$ is not a maximal antipodal subset in $P_{4}(8)$. Of course $A(4,8)$ is not maximal in $P_{4}(9)$. 
In order to state the main theorem of this section we prepare some notation. A subset $A \subset P_{k}(n)$ is said to be full in $P_{k}(n)$, if $A$ is not congruent with any subset of $P_{k}(n-1)$. For example $B(4,7)$ is full in $P_{4}(7)$, but it is not full in $P_{4}(8)$. We denote

$$
A+m=\{\{\alpha(1)+m, \ldots, \alpha(k)+m\} \mid \alpha \in A\}
$$

for an integer $m$.

The following main theorem of this section states that any maximal antipodal subset of $P_{4}(n)$ is described by $A(4,2 l), B(4,7)$ and $B(4,8)$.

Theorem 6.1. Each of the followings is a maximal antipodal subset of $P_{4}(n)$ :

\begin{tabular}{|c|c|c|c|c|c|}
\hline$n$ & 4,5 & 6 & 7 & 8,9 & 10 \\
\hline & $A(4,4)$ & $A(4,6)$ & $B(4,7)$ & $B(4,8)$ & $A(4,10), B(4,8)$ \\
\hline
\end{tabular}

In the case $n>10, A(4,2[n / 2]), B(4,7) \cup\left[\left(\right.\right.$ a full MAS in $\left.P_{4}(n-7)\right)+$ 7] and $B(4,8) \cup\left[\left(a M A S\right.\right.$ in $\left.\left.P_{4}(n-8)\right)+8\right]$, where $M A S$ is an abbreviation of maximal antipodal subset. Conversely any maximal antipodal subset of $P_{4}(n)$ is congruent with one of them.

Proof. We prove the theorem in each cases for $n \leq 10$ and by induction on $n$ for $n>10$. We take $A_{1}=\{\{1,2,3,4\}\}$. We have

$$
A\left(A_{1}\right)=P_{2}(\{1,2,3,4\}) \times P_{2}(\{5, \ldots, n\}) \cup P_{4}(\{5, \ldots, n\}) .
$$

If $n \leq 5, A\left(A_{1}\right)$ is empty and $A_{1}=A(4,4)$ is a maximal antipodal subset of $P_{4}(n)$.

Thus we suppose that $n \geq 6$. The stabilizer $S\left(A_{1}\right)$ is equal to $\operatorname{Sym}(\{1,2,3,4\}) \times \operatorname{Sym}(\{5, \ldots, n\})$, which acts transitively on

$$
P_{2}(\{1,2,3,4\}) \times P_{2}(\{5, \ldots, n\}) \text { and } P_{4}(\{5, \ldots, n\}) .
$$

These are two orbits of $S\left(A_{1}\right)$. Hence we divide the procedure to two cases where we take minimum elements in the two orbits.

(1) We take the minimum element $\{1,2,5,6\}$ of the orbit $P_{2}(\{1,2,3,4\}) \times$ $P_{2}(\{5, \ldots, n\})$, add it to $A_{1}$ and obtain $A_{2,1}=\{\{1,2,3,4\},\{1,2,5,6\}\}$. 
We have

$$
\begin{aligned}
A\left(A_{2,1}\right)= & \left\{\alpha \in A\left(A_{1}\right)-\{\{1,2,5,6\}\} \mid \alpha \text { and }\{1,2,5,6\} \text { are antipodal }\right\} \\
= & \{\{3,4,5,6\}\} \cup\{\{1,2\},\{3,4\}\} \times P_{2}(\{7, \ldots, n\}) \\
& \cup P_{1}(\{1,2\}) \times P_{1}(\{3,4\}) \times P_{1}(\{5,6\}) \times P_{1}(\{7, \ldots, n\}) \\
& \cup P_{2}(\{5,6\}) \times P_{2}(\{7, \ldots, n\}) \cup P_{4}(\{7, \ldots, n\}) \\
= & \{\{3,4,5,6\}\} \\
& \cup P_{1}(\{1,2\}) \times P_{1}(\{3,4\}) \times P_{1}(\{5,6\}) \times P_{1}(\{7, \ldots, n\}) \\
& \cup\{\{1,2\},\{3,4\},\{5,6\}\} \times P_{2}(\{7, \ldots, n\}) \\
& \cup P_{4}(\{7, \ldots, n\}) .
\end{aligned}
$$

In $A\left(A_{2,1}\right),\{3,4,5,6\}$ and other elements are antipodal. We can add it to $A_{2,1}$ and obtain

$$
A_{3,1}=\{\{1,2,3,4\},\{1,2,5,6\},\{3,4,5,6\}\}=A(4,6) .
$$

We have

$$
\begin{aligned}
A\left(A_{3,1}\right)= & P_{1}(\{1,2\}) \times P_{1}(\{3,4\}) \times P_{1}(\{5,6\}) \times P_{1}(\{7, \ldots, n\}) \\
& \cup\{\{1,2\},\{3,4\},\{5,6\}\} \times P_{2}(\{7, \ldots, n\}) \\
& \cup P_{4}(\{7, \ldots, n\}) .
\end{aligned}
$$

The stabilizer $S\left(A_{3,1}\right)$ induces permutations on $\{\{1,2\},\{3,4\},\{5,6\}\}$, so it acts transitively on each of

$$
\begin{aligned}
& P_{1}(\{1,2\}) \times P_{1}(\{3,4\}) \times P_{1}(\{5,6\}) \times P_{1}(\{7, \ldots, n\}), \\
& \{\{1,2\},\{3,4\},\{5,6\}\} \times P_{2}(\{7, \ldots, n\}), \\
& P_{4}(\{7, \ldots, n\}) .
\end{aligned}
$$

These are three orbits of $S\left(A_{3,1}\right)$. In the case $n=6$, we have $A\left(A_{3,1}\right)=$ $\emptyset$ and $A_{3,1}$ is a maximal antipodal subset of $P_{4}(6)$. Thus we suppose that $n \geq 7$. We divide the procedure to the three cases.

(1.1) We take the minimum element $\{1,3,5,7\}$ of the orbit $P_{1}(\{1,2\}) \times$ $P_{1}(\{3,4\}) \times P_{1}(\{5,6\}) \times P_{1}(\{7, \ldots, n\})$, add it to $A_{3,1}$ and obtain

$$
A_{4,1}=\{\{1,2,3,4\},\{1,2,5,6\},\{3,4,5,6\},\{1,3,5,7\}\} .
$$

We have

$$
\begin{aligned}
A\left(A_{4,1}\right)= & \left\{\alpha \in A\left(A_{3,1}\right)-\{\{1,3,5,7\}\} \mid \alpha \text { and }\{1,3,5,7\} \text { are antipodal }\right\} \\
= & \{\{1,4,6,7\},\{2,3,6,7\},\{2,4,5,7\}\} \\
& \cup\{\{1,3,6\},\{1,4,5\},\{2,3,5\},\{2,4,6\}\} \times P_{1}(\{8, \ldots, n\}) \\
& \cup\{\{1,2,7\},\{3,4,7\},\{5,6,7\}\} \times P_{1}(\{8, \ldots, n\}) \\
& \cup P_{4}(\{8, \ldots, n\}) .
\end{aligned}
$$


Any element of $\{\{1,4,6,7\},\{2,3,6,7\},\{2,4,5,7\}\}$ and any element of $A\left(A_{4,1}\right)$ are antipodal. We can add these three elements to $A_{4,1}$ and obtain

$$
\begin{aligned}
A_{7,1}= & \{\{1,2,3,4\},\{1,2,5,6\},\{3,4,5,6\},\{1,3,5,7\}, \\
& \{1,4,6,7\},\{2,3,6,7\},\{2,4,5,7\}\} .
\end{aligned}
$$

From the description of $A_{7,1}$ we get

$$
\begin{aligned}
A_{7,1}^{c}= & \left\{\alpha^{c} \mid \alpha \in A_{7,1}\right\} \\
= & \{\{5,6,7\},\{3,4,7\},\{1,2,7\},\{2,4,6\}, \\
& \{2,3,5\},\{1,4,5\},\{1,3,6\}\} .
\end{aligned}
$$

Acting the permutation $\left(\begin{array}{lllllll}1 & 2 & 3 & 4 & 5 & 6 & 7 \\ 7 & 6 & 5 & 4 & 3 & 2 & 1\end{array}\right)$ on $A_{7,1}^{c}$ we obtain $B(3,7)$. So $A_{7,1}$ and $B(4,7)=B^{c}(3,7)$ are congruent. We have

$$
\begin{aligned}
A\left(A_{7,1}\right)= & \{\{1,2,7\},\{3,4,7\},\{5,6,7\}, \\
& \{1,3,6\},\{1,4,5\},\{2,3,5\},\{2,4,6\}\} \times P_{1}(\{8, \ldots, n\}) \\
& \cup P_{4}(\{8, \ldots, n\}) \\
= & A_{7,1}^{c} \times P_{1}(\{8, \ldots, n\}) \cup P_{4}(\{8, \ldots, n\}) .
\end{aligned}
$$

Since the group of all projective transformations on $F_{2} P^{2}$ acts transitively on the set of all projective lines in it, the stabilizer $S\left(A_{7,1}\right)$ acts transitively on $A_{7,1}^{c} \times P_{1}(\{8, \ldots, n\})$. It also acts transitively on $P_{4}(\{8, \ldots, n\})$. We can also directly see that $S\left(A_{7,1}\right)$ acts transitively on $A_{7,1}^{c} \times P_{1}(\{8, \ldots, n\})$. Hence

$$
A_{7,1}^{c} \times P_{1}(\{8, \ldots, n\}), \quad P_{4}(\{8, \ldots, n\})
$$

are two orbits of $S\left(A_{7,1}\right)$. In the case $n=7$ we have $A\left(A_{7,1}\right)=\emptyset$ and $A_{7,1}$ is a maximal antipodal subset of $P_{4}(7)$. Thus we suppose that $n \geq 8$ in the case (1.1).

(1.1.1) We take the minimum element $\{1,2,7,8\}$ of the orbit $A_{7,1}^{c} \times$ $P_{1}(\{8, \ldots, n\})$, add it to $A_{7,1}$ and obtain

$$
\begin{aligned}
A_{8,1}= & \{\{1,2,3,4\},\{1,2,5,6\},\{3,4,5,6\},\{1,3,5,7\}, \\
& \{1,4,6,7\},\{2,3,6,7\},\{2,4,5,7\},\{1,2,7,8\}\} .
\end{aligned}
$$

We have

$$
\begin{aligned}
A( & \left.A_{8,1}\right) \\
= & \{\{3,4,7\},\{5,6,7\},\{1,3,6\},\{1,4,5\},\{2,3,5\},\{2,4,6\}\} \times\{\{8\}\} \\
& \cup P_{4}(\{9, \ldots, n\}) .
\end{aligned}
$$


$\{\{3,4,7\},\{5,6,7\},\{1,3,6\},\{1,4,5\},\{2,3,5\},\{2,4,6\}\} \times\{\{8\}\}$ is antipodal and any element of it and any element of $A\left(A_{8,1}\right)$ are antipodal, so we can add these six elements to $A_{8,1}$ and obtain

$$
\begin{aligned}
A_{14}= & \{\{1,2,3,4\},\{1,2,5,6\},\{3,4,5,6\},\{1,3,5,7\}, \\
& \{1,4,6,7\},\{2,3,6,7\},\{2,4,5,7\}\} \\
& \cup\{\{1,2,7\},\{3,4,7\},\{5,6,7\},\{1,3,6\}, \\
& \{1,4,5\},\{2,3,5\},\{2,4,6\}\} \times\{\{8\}\} \\
= & A_{7,1} \cup A_{7,1}^{c} \times\{\{8\}\},
\end{aligned}
$$

which is congruent with $B(4,8)$. We have

$$
A\left(A_{14}\right)=P_{4}(\{9, \ldots, n\}),
$$

which is equivalent to $A(B(4,8))=P_{4}(\{9, \ldots, n\})$. In the cases $n=$ $8,9,10,11 A\left(A_{14}\right)$ is empty and $A_{14}$ is a maximal antipodal subset of $P_{4}(n)$. In the case $n>11$ any maximal antipodal subset of $P_{4}(n)$ we reach in this case is equal to $A_{14} \cup B$ for a maximal antipodal subset $B$ of $P_{4}(\{9, \ldots, n\})$.

(1.1.2) We take the minimum element $\{8,9,10,11\}$ of the orbit $P_{4}(\{8, \ldots, n\})$, add it to $A_{7,1}$ and obtain

$$
\begin{aligned}
A_{8,2}= & \{\{1,2,3,4\},\{1,2,5,6\},\{3,4,5,6\},\{1,3,5,7\}, \\
& \{1,4,6,7\},\{2,3,6,7\},\{2,4,5,7\},\{8,9,10,11\}\} .
\end{aligned}
$$

We have

$$
\begin{aligned}
A\left(A_{8,2}\right)= & A_{7,1}^{c} \times P_{1}(\{12, \ldots, n\}) \\
& \cup P_{2}(\{8,9,10,11\}) \times P_{2}(\{12, \ldots, n\}) \cup P_{4}(\{12, \ldots, n\}) .
\end{aligned}
$$

The stabilizer $S\left(A_{8,2}\right)$ is equal to

$$
S\left(A_{7,1}^{c}\right) \times \operatorname{Sym}(\{8,9,10,11\}) \times \operatorname{Sym}(\{12, \ldots, n\}),
$$

which acts transitively on each of

$$
\begin{aligned}
& A_{7,1}^{c} \times P_{1}(\{12, \ldots, n\}), \\
& P_{2}(\{8,9,10,11\}) \times P_{2}(\{12, \ldots, n\}), \\
& P_{4}(\{12, \ldots, n\}) .
\end{aligned}
$$

These are three orbits of $S\left(A_{8,2}\right)$. We divide the procedure to two cases of (1.1.2.1) $A_{7,1}^{c} \times P_{1}(\{12, \ldots, n\})$ and (1.1.2.2) $P_{2}(\{8,9,10,11\}) \times$ $P_{2}(\{12, \ldots, n\}), P_{4}(\{12, \ldots, n\})$.

(1.1.2.1) In the case $n \geq 12$ we take the minimum element $\{1,2,7,12\}$ of the orbit $A_{7,1}^{c} \times P_{1}(\{12, \ldots, n\})$. In a way similar to the case (1.1.1) we reach the union of a maximal antipodal subset in $P_{4}(\{1, \ldots, 7\} \cup$ 
$\{12\})$ which is congruent with $B(4,8)$ and a maximal antipodal subset of

$$
P_{4}(\{8,9,10,11\} \cup\{13, \ldots, n\}) .
$$

This is congruent with the union of $B(4,8)$ and a maximal antipodal subset in $P_{4}(\{9, \ldots, n\})$.

(1.1.2.2) We take the minimum element of the orbit $P_{2}(\{8,9,10,11\}) \times$ $P_{2}(\{12, \ldots, n\}), P_{4}(\{12, \ldots, n\})$ and repeat the procedure. If we take an element of $A_{7,1}^{c} \times P_{1}(\{12, \ldots, n\})$ in this case, then the result reduces to the case (1.1.2.1). Hence it is sufficient to consider maximal antipodal subsets of $P_{4}(n)$ which are included in

$$
\begin{aligned}
& A_{8,2} \cup P_{2}(\{8,9,10,11\}) \times P_{2}(\{12, \ldots, n\}) \cup P_{4}(\{12, \ldots, n\}) \\
& \subset A_{7,1} \cup P_{4}(\{8, \ldots, n\}) .
\end{aligned}
$$

Any maximal antipodal subset of $P_{4}(n)$ we reach in this case is equal to $A_{7,1} \cup B$ for a maximal antipodal subset of $P_{4}(\{8, \ldots, n\})$. If $B$ is not full in $P_{4}(\{8, \ldots, n\})$, then there exists $m \in\{8, \ldots, n\}$ which is not contained in any element of $B$ and

$$
A_{7,1} \cup B \subset A_{7,1} \cup\left(A_{7,1}^{c} \times\{\{m\}\}\right) \cup B .
$$

Here $A_{7,1} \cup\left(A_{7,1}^{c} \times\{\{m\}\}\right) \cup B$ is antipodal and this contradicts to the maximal property of $A_{7,1} \cup B$. Thus $B$ is full in $P_{4}(\{8, \ldots, n\})$. Conversely we show that $A_{7,1} \cup B$ is a maximal antipodal subset of $P_{4}(n)$ if $B$ is a full maximal antipodal subset of $P_{4}(\{8, \ldots, n\})$. If $A_{7,1} \cup B$ is not a maximal antipodal subset of $P_{4}(n)$, then there exists $\alpha \notin A_{7,1} \cup B$ such that $\alpha$ and any element of $A_{7,1} \cup B$ are antipodal. In particular

$$
\alpha \in A\left(A_{7,1}\right)=A_{7,1}^{c} \times P_{1}(\{8, \ldots, n\}) \cup P_{4}(\{8, \ldots, n\}) .
$$

If $\alpha$ belongs to $P_{1}(\{8, \ldots, n\})$, then this contradicts the maximal property of $B$. If $\alpha$ belongs to $A_{7,1}^{c} \times P_{1}(\{8, \ldots, n\})$, then $\#(\alpha \cap\{8, \ldots, n\})=$ 1. By the assumption of induction $B$ is a certain union of copies of $B(4,7), B(4,8)$ and $A(4,2 l)$. Moreover $B$ is full in $P_{4}(\{8, \ldots, n\})$. So there exists an element $\beta$ in $B$ such that $\#(\alpha \cap \beta)=1$, which is a contradiction. Therefore $A_{7,1} \cup B$ is a maximal antipodal subset of $P_{4}(n)$.

(1.2) We take the minimum element $\{1,2,7,8\}$ of the orbit

$$
\{\{1,2\},\{3,4\},\{5,6\}\} \times P_{2}(\{7, \ldots, n\}),
$$

add it to $A_{3,1}=A(4,6)$ and obtain

$$
A_{4,2}=A(4,6) \cup\{\{1,2,7,8\}\} .
$$


We have

$$
\begin{aligned}
A\left(A_{4,2}\right)= & P_{1}(\{1,2\}) \times P_{1}(\{3,4\}) \times P_{1}(\{5,6\}) \times P_{1}(\{7,8\}) \\
& \cup\{\{3,4,7,8\},\{5,6,7,8\}\} \\
& \cup\{\{1,2\},\{3,4\},\{5,6\}\} \times P_{2}(\{9, \ldots, n\}) \\
& \cup P_{2}(\{7,8\}) \times P_{2}(\{9, \ldots, n\}) \cup P_{4}(\{9, \ldots, n\}) .
\end{aligned}
$$

$\{\{3,4,7,8\},\{5,6,7,8\}\}$ is antipodal and any element of it and any element of $A\left(A_{4,2}\right)$ are antipodal, so we can add it to $A_{4,2}$ and obtain

$$
A_{6,1}=A(4,6) \cup\{\{1,2,7,8\},\{3,4,7,8\},\{5,6,7,8\}\}=A(4,8) .
$$

We have

$$
\begin{aligned}
A\left(A_{6,1}\right)= & P_{1}(\{1,2\}) \times P_{1}(\{3,4\}) \times P_{1}(\{5,6\}) \times P_{1}(\{7,8\}) \\
& \cup\{\{1,2\},\{3,4\},\{5,6\},\{7,8\}\} \times P_{2}(\{9, \ldots, n\}) \\
& \cup P_{4}(\{9, \ldots, n\}) .
\end{aligned}
$$

The stabilizer $A\left(A_{6,1}\right)$ induces permutations on

$$
\{\{1,2\},\{3,4\},\{5,6\},\{7,8\}\},
$$

so it acts transitively on each of

$$
\begin{aligned}
& P_{1}(\{1,2\}) \times P_{1}(\{3,4\}) \times P_{1}(\{5,6\}) \times P_{1}(\{7,8\}), \\
& \{\{1,2\},\{3,4\},\{5,6\},\{7,8\}\} \times P_{2}(\{9, \ldots, n\}), \\
& P_{4}(\{9, \ldots, n\}) .
\end{aligned}
$$

These are three orbits of $S\left(A_{6,1}\right)$. Hence we divide the procedure to three cases.

(1.2.1) We take the minimum element $\{1,3,5,7\}$ of the orbit

$$
P_{1}(\{1,2\}) \times P_{1}(\{3,4\}) \times P_{1}(\{5,6\}) \times P_{1}(\{7,8\}),
$$

add it to $A_{6,1}$ and obtain

$$
A_{7,2}=A(4,8) \cup\{\{1,3,5,7\}\} .
$$

Let

$$
\begin{aligned}
E v_{8}=\{ & \left\{i_{1}, i_{2}, i_{3}, i_{4}\right\} \mid i_{j} \in\{2 j-1,2 j\}(1 \leq j \leq 4), \\
& \text { the number of even numbers is even }\}
\end{aligned}
$$

We have

$$
A\left(A_{7,2}\right)=\left(E v_{8}-\{1,3,5,7\}\right) \cup P_{4}(\{9, \ldots, n\}) .
$$

Any element of $E v_{8}$ and any element of $A\left(A_{7,2}\right)$ are antipodal, so we can add it to $A_{7,2}$ and obtain

$$
A(4,8) \cup E v_{8}=A_{14}
$$


Thus the procedure in this case reduces to the case (1.1.1).

(1.2.2) We take the minimum element $\{1,2,9,10\}$ of the orbit

$$
\{\{1,2\},\{3,4\},\{5,6\},\{7,8\}\} \times P_{2}(\{9, \ldots, n\}),
$$

add it to $A_{6,1}$ and obtain

$$
A_{7,3}=A(4,8) \cup\{\{1,2,9,10\}\} .
$$

We have

$$
\begin{aligned}
A\left(A_{7,3}\right)= & \{\{3,4,9,10\},\{5,6,9,10\},\{7,8,9,10\}\} \\
& \cup\{\{1,2\},\{3,4\},\{5,6\},\{7,8\}\} \times P_{2}(\{11, \ldots, n\}) \\
& \cup P_{2}(\{9,10\}) \times P_{2}(\{11, \ldots, n\}) \\
& \cup P_{4}(\{11, \ldots, n\}) .
\end{aligned}
$$

$\{\{3,4,9,10\},\{5,6,9,10\},\{7,8,9,10\}\}$ is antipodal. Any element of it and any element of $A\left(A_{7,3}\right)$ are antipodal, so we can add it to $A_{7,3}$ and obtain

$$
\begin{aligned}
A_{10} & =A(4,8) \cup\{\{1,2,9,10\},\{3,4,9,10\},\{5,6,9,10\},\{7,8,9,10\}\} \\
& =A(4,10) .
\end{aligned}
$$

We have

$$
\begin{aligned}
A\left(A_{10}\right)= & \{\{1,2\},\{3,4\},\{5,6\},\{7,8\},\{9,10\}\} \times P_{2}(\{11, \ldots, n\}) \\
& \cup P_{4}(\{11, \ldots, n\}) .
\end{aligned}
$$

Let $M$ be a maximal antipodal subset of $P_{4}(n)$ we reach in this case. Since $M-A_{10} \subset A\left(A_{10}\right)$, there exist

$$
\begin{aligned}
& M_{1} \subset\{\{1,2\},\{3,4\},\{5,6\},\{7,8\},\{9,10\}\} \times P_{2}(\{11, \ldots, n\}), \\
& M_{2} \subset P_{4}(\{11, \ldots, n\})
\end{aligned}
$$

satisfying $M=A_{10} \cup M_{1} \cup M_{2}$. Let

$$
\begin{aligned}
N_{1}=\left\{\beta \in P_{2}(\{11, \ldots, n\}) \mid\right. & \alpha \cup \beta \in M_{1} \text { for some } \\
& \alpha=\{2 j-1,2 j\}(1 \leq j \leq 5)\} .
\end{aligned}
$$

This is an antipodal subset of $P_{2}(\{11, \ldots, n\})$. So by an action of $\operatorname{Sym}(\{11, \ldots, n\})$ we can suppose that

$$
N_{1}=\{\{11,12\},\{13,14\}, \ldots,\{2 m-1,2 m\}\} .
$$

Any element of $N_{1}$ and any element of $M_{2}$ have even intersection. Hence any element of $A(4,2 \mathrm{~m})$ and any element of $M_{2}$ are antipodal and $A(4,2 m) \cup M_{2}$ is antipodal. We have

$$
M=A_{10} \cup M_{1} \cup M_{2} \subset A(4,2 m) \cup M_{2} .
$$


Since $M$ is a maximal antipodal subset of $P_{4}(n)$, we obtain $M=$ $A(4,2 m) \cup M_{2}$. By repeating the procedure adding $\{1,2,11,12\},\{1,2,13,14\}$ and so on, we reach $A(4,2 m)$ and have

$$
\begin{aligned}
A(A(4,2 m))= & \{\{1,2\}, \ldots,\{2 m-1,2 m\}\} \times P_{2}(\{2 m+1, \ldots, n\}) \\
& \cup P_{4}(\{2 m+1, \ldots, n\}) .
\end{aligned}
$$

Thus there exist

$$
\begin{aligned}
& M_{3} \subset\{\{1,2\}, \ldots,\{2 m-1,2 m\}\} \times P_{2}(\{2 m+1, \ldots, n\}), \\
& M_{4} \subset P_{4}(\{2 m+1, \ldots, n\})
\end{aligned}
$$

satisfying $M=A(4,2 m) \cup M_{3} \cup M_{4}$. If $M_{3}$ is not empty, then there exists $\{2 a-1,2 a, b, c\}$ with $1 \leq a \leq m, 2 m+1 \leq b, c$. The element $\{b, c\}$ of $P_{2}(\{2 m+1, \ldots, n\})$ and any element of $M_{4}$ have even intersection. Hence $\{1,2, b, c\}$ and any element of $M_{4}$ are antipodal and $\{b, c\}$ belongs to $N_{1}$, which is a contradiction. Therefore $M_{3}$ is empty and $M=A(4,2 m) \cup M_{4}$. So $M_{4}$ is a maximal antipodal subset of $P_{4}(\{2 m+1, \ldots, n\})$. By the assumption of induction $M_{4}$ is a certain disjoint union of copies of $B(4,3), B(4,8)$ and at most $A(4,2 l)+2 m$. If there exists $A(4,2 l)+2 m$, then

$$
A(4,2 m) \cup(A(4,2 l)+2 m) \subset A(4,2(m+l))
$$

and moreover any element of $A(4,2 l)+2 m$ and any element of $M_{4}$. So any element of $A(4,2(m+l))$ and any element of $M_{4}$ are antipodal, too. Hence $A(4,2(m+l)) \cup M_{4}$ is antipodal and

$$
M=A(4,2 m) \cup(A(4,2 l)+2 m) \cup M_{4} \subset A(4,2(m+l)) \cup M_{4},
$$

which contradicts to the maximality of $M$. So $M_{4}$ is a certain disjoint union of copies of $B(4,7)$ and $B(4,8)$. If $M_{4}$ is empty, then $M=$ $A(4,2 m)$. If $M_{4}$ is not empty, then $M$ includes $B(4,7)$ or $B(4,8)$. Thus the procedure in this case reduces to the case (1.1.2.2) or (1.1.1). (1.2.3) We take the minimum element $\{9,10,11,12\}$ of the orbit $P_{4}(\{9, \ldots, n\})$, add it to $A_{6,1}$ and obtain

$$
A_{7,4}=A(4,8) \cup\{\{9,10,11,12\}\} .
$$

We have

$$
\begin{aligned}
A\left(A_{7,4}\right)= & P_{1}(\{1,2\}) \times P_{1}(\{3,4\}) \times P_{1}(\{5,6\}) \times P_{1}(\{7,8\}) \\
& \cup\{\{1,2\},\{3,4\},\{5,6\},\{7,8\}\} \times P_{2}(\{9,10,11,12\}) \\
& \cup\{\{1,2\},\{3,4\},\{5,6\},\{7,8\}\} \times P_{2}(\{13, \ldots, n\}) \\
& \cup P_{2}(\{9,10,11,12\}) \times P_{2}(\{13, \ldots, n\}) \\
& \cup P_{4}(\{13, \ldots, n\}) .
\end{aligned}
$$


The stabilizer $S\left(A_{7,4}\right)$ induces permutations on $\{\{1,2\},\{3,4\},\{5,6\},\{7,8\}\}$ and $\{9,10,11,12\}$, so it transitively acts on each of

$$
\begin{aligned}
& P_{1}(\{1,2\}) \times P_{1}(\{3,4\}) \times P_{1}(\{5,6\}) \times P_{1}(\{7,8\}), \\
& \{\{1,2\},\{3,4\},\{5,6\},\{7,8\}\} \times P_{2}(\{9,10,11,12\}), \\
& \{\{1,2\},\{3,4\},\{5,6\},\{7,8\}\} \times P_{2}(\{13, \ldots, n\}), \\
& P_{2}(\{9,10,11,12\}) \times P_{2}(\{13, \ldots, n\}), \\
& P_{4}(\{13, \ldots, n\}) .
\end{aligned}
$$

These are five orbits of $S\left(A_{7,4}\right)$. Hence we divide the procedure to five cases.

(1.2.3.1) We take the minimum element $\{1,3,5,7\}$ of the orbit $P_{1}(\{1,2\}) \times$ $P_{1}(\{3,4\}) \times P_{1}(\{5,6\}) \times P_{1}(\{7,8\})$. This reduces to the case (1.1). (1.2.3.2) We take the minimum element $\{1,2,9,10\}$ of the orbit

$$
\{\{1,2\},\{3,4\},\{5,6\},\{7,8\}\} \times P_{2}(\{9,10,11,12\}) .
$$

This reduces to the case (1.2.2).

(1.2.3.3) We take the minimum element $\{1,2,13,14\}$ of the orbit

$$
\{\{1,2\},\{3,4\},\{5,6\},\{7,8\}\} \times P_{2}(\{13, \ldots, n\}) .
$$

This reduces to the case (1.2.2) by the action of the permutation (9 13) (10 14).

(1.2.3.4) We consider the orbits of $P_{2}(\{9,10,11,12\}) \times P_{2}(\{13, \ldots, n\})$ and $P_{4}(\{13, \ldots, n\})$. When we proceed the procedure in this case, if we take one element of the orbits of (1.2.3.1), (1.2.3.2) and (1.2.3.3), this reduces to the cases of (1.1) or (1.2.2). Thus it is sufficient to take elements of $P_{2}(\{9,10,11,12\}) \times P_{2}(\{13, \ldots, n\})$ and $P_{4}(\{13, \ldots, n\})$. For any maximal antipodal subset $M$ we reach in this case there exists an antipodal subset $M_{1}$ of $P_{4}(\{9, \ldots, n\})$ which satisfies $M=A(4,8) \cup$ $M_{1}$. However, this does not happen, because $A(4,8)$ is not a maximal antipodal subset of $P_{4}(8)$.

(1.3) We take the minimum element $\{7,8,9,10\}$ of the orbit $P_{4}(\{7, \ldots, n\})$, add it to $A_{3,1}$ and obtain

$$
A_{4,3}=A(4,6) \cup\{\{7,8,9,10\}\} .
$$

We have

$$
\begin{aligned}
A\left(A_{4,3}\right)= & P_{1}(\{1,2\}) \times P_{1}(\{3,4\}) \times P_{1}(\{5,6\}) \times P_{1}(\{11, \ldots, n\}) \\
& \cup\{\{1,2\},\{3,4\},\{5,6\}\} \times P_{2}(\{7,8,9,10\}) \\
& \cup\{\{1,2\},\{3,4\},\{5,6\}\} \times P_{2}(\{11, \ldots, n\}) \\
& \cup P_{2}(\{7,8,9,10\}) \times P_{2}(\{11, \ldots, n\}) \\
& \cup P_{4}(\{11, \ldots, n\}) .
\end{aligned}
$$


The stabilizer $S\left(A_{4,3}\right)$ induces permutations on $\{\{1,2\},\{3,4\},\{5,6\}\}$ and $\{7,8,9,10\}$, so it acts transitively on each of

$$
\begin{aligned}
& P_{1}(\{1,2\}) \times P_{1}(\{3,4\}) \times P_{1}(\{5,6\}) \times P_{1}(\{11, \ldots, n\}), \\
& \{\{1,2\},\{3,4\},\{5,6\}\} \times P_{2}(\{7,8,9,10\}), \\
& \{\{1,2\},\{3,4\},\{5,6\}\} \times P_{2}(\{11, \ldots, n\}), \\
& P_{2}(\{7,8,9,10\}) \times P_{2}(\{11, \ldots, n\}), \\
& P_{4}(\{11, \ldots, n\}) .
\end{aligned}
$$

These are five orbits of $S\left(A_{4,3}\right)$. Hence we divide the procedure to five cases.

(1.3.1) We take the minimum element $\{1,3,5,11\}$ of the orbit $P_{1}(\{1,2\}) \times$ $P_{1}(\{3,4\}) \times P_{1}(\{5,6\}) \times P_{1}(\{11, \ldots, n\})$ and add it to $A_{4,3}$. This case reduces to the case (1.1) by the action of the permutation $(711)(812)$. (1.3.2) We take the minimum element $\{1,2,7,8\}$ of the orbit

$$
\{\{1,2\},\{3,4\},\{5,6\}\} \times P_{2}(\{7,8,9,10\})
$$

and add it to $A_{4,3}$. This case reduces to the case (1.2).

(1.3.3) We take the minimum element $\{1,2,11,12\}$ of the orbit

$$
\{\{1,2\},\{3,4\},\{5,6\}\} \times P_{2}(\{11, \ldots, n\})
$$

and add it to $A_{4,3}$. This case reduces to the case (1.2) by the action of the permutation $(711)(812)$.

(1.3.4) We take the minimum element $\{7,8,11,12\}$ of the orbit

$$
P_{2}(\{7,8,9,10\}) \times P_{2}(\{11, \ldots, n\}),
$$

add it to $A_{4,3}$ and obtain

$$
A_{5}=A(4,6) \cup\{\{7,8,9,10\},\{7,8,11,12\}\} .
$$

We have

$$
\begin{aligned}
A\left(A_{5}\right)= & P_{1}(\{1,2\}) \times P_{1}(\{3,4\}) \times P_{1}(\{5,6\}) \times P_{1}(\{13, \ldots, n\}) \\
& \cup\{\{1,2\},\{3,4\},\{5,6\}\} \times\{\{7,8\},\{9,10\},\{11,12\}\} \\
& \cup\{\{1,2\},\{3,4\},\{5,6\}\} \times P_{2}(\{13, \ldots, n\}) \\
& \cup\{\{9,10,11,12\}\} \\
& \cup\{\{7,8\},\{9,10\},\{11,12\}\} \times P_{2}(\{13, \ldots, n\}) \\
& \cup P_{1}(\{7,8\}) \times P_{1}(\{9,10\}) \times P_{1}(\{11,12\}) \times P_{1}(\{13, \ldots, n\}) \\
& \cup P_{4}(\{13, \ldots, n\}) .
\end{aligned}
$$

In $A\left(A_{5}\right)$ the element $\{9,10,11,12\}$ and other elements are antipodal, so we can add it to $A_{5}$ and obtain

$$
A_{6,2}=A(4,6) \cup(A(4,6)+6) .
$$


We have

$$
\begin{aligned}
A\left(A_{6,2}\right)= & P_{1}(\{1,2\}) \times P_{1}(\{3,4\}) \times P_{1}(\{5,6\}) \times P_{1}(\{13, \ldots, n\}) \\
& \cup\{\{1,2\},\{3,4\},\{5,6\}\} \times\{\{7,8\},\{9,10\},\{11,12\}\} \\
& \cup\{\{1,2\},\{3,4\},\{5,6\},\{7,8\},\{9,10\},\{11,12\}\} \times P_{2}(\{13, \ldots, n\}) \\
& \cup P_{1}(\{7,8\}) \times P_{1}(\{9,10\}) \times P_{1}(\{11,12\}) \times P_{1}(\{13, \ldots, n\}) \\
& \cup P_{4}(\{13, \ldots, n\}) .
\end{aligned}
$$

The stabilizer $S\left(A_{6,2}\right)$ induces permutations on $\{\{1,2\},\{3,4\},\{5,6\}\}$ and $\{\{7,8\},\{9,10\},\{11,12\}\}$ and interchanging these two, so it acts transitively on each of

$$
\begin{aligned}
& P_{1}(\{1,2\}) \times P_{1}(\{3,4\}) \times P_{1}(\{5,6\}) \times P_{1}(\{13, \ldots, n\}) \\
& \cup P_{1}(\{7,8\}) \times P_{1}(\{9,10\}) \times P_{1}(\{11,12\}) \times P_{1}(\{13, \ldots, n\}), \\
& \{\{1,2\},\{3,4\},\{5,6\}\} \times\{\{7,8\},\{9,10\},\{11,12\}\}, \\
& \{\{1,2\},\{3,4\},\{5,6\},\{7,8\},\{9,10\},\{11,12\}\} \times P_{2}(\{13, \ldots, n\}) \\
& P_{4}(\{13, \ldots, n\}) .
\end{aligned}
$$

These are four orbits of $S\left(A_{6,2}\right)$. Hence we divide the procedure to four cases.

(1.3.4.1) We take the minimum element $\{1,3,5,13\}$ of the orbit

$$
\begin{aligned}
& P_{1}(\{1,2\}) \times P_{1}(\{3,4\}) \times P_{1}(\{5,6\}) \times P_{1}(\{13, \ldots, n\}) \\
& \cup P_{1}(\{7,8\}) \times P_{1}(\{9,10\}) \times P_{1}(\{11,12\}) \times P_{1}(\{13, \ldots, n\})
\end{aligned}
$$

and add it to $A_{6,2}$. This reduces to the case (1.1).

(1.3.4.2) We take the minimum element $\{1,2,7,8\}$ of the orbit

$$
\{\{1,2\},\{3,4\},\{5,6\}\} \times\{\{7,8\},\{9,10\},\{11,12\}\}
$$

and add it to $A_{6,2}$. This reduces to the case (1.3.2).

(1.3.4.3) We take the minimum element $\{1,2,13,14\}$ of the orbit

$$
\begin{aligned}
& \{\{1,2\},\{3,4\},\{5,6\}\} \times P_{2}(\{13, \ldots, n\}) \\
& \cup\{\{7,8\},\{9,10\},\{11,12\}\} \times P_{2}(\{13, \ldots, n\})
\end{aligned}
$$

and add it to $A_{6,2}$. This reduces to the case (1.2).

(1.3.4.4) We consider the orbit $P_{4}(\{13, \ldots, n\})$. When we proceed the procedure in this case, if we take one element of the orbits of (1.3.4.1), (1.3.4.2) and (1.3.4.3), this reduces to the cases of (1.1), (1.2) or (1.3.2). Thus it is sufficient to take elements of $\{\{1,2\},\{3,4\},\{5,6\}\} \times P_{2}(\{13, \ldots, n\})$ and $\{\{1,2\},\{3,4\},\{5,6\}\} \times P_{2}(\{13, \ldots, n\})$. For any maximal antipodal subset $M$ we reach in this case there exists an antipodal subset $M_{1}$ of $P_{4}(\{13, \ldots, n\})$ which satisfies $M=A_{6,2} \cup M_{1}$. However, this does 
not happen, because $A_{6,2}=A(4,6) \cup(A(4,6)+6)$ is not a maximal antipodal subset of $P_{4}(12)$.

(1.3.5) We consider the orbit $P_{4}(\{11, \ldots, n\})$. When we proceed the procedure in this case, if we take one element of the orbits of (1.3.1), (1.3.2), (1.3.3) and (1.3.4), this reduces to the cases already mentioned above. Thus it is sufficient to take elements of $P_{4}(\{13, \ldots, n\})$. For any maximal antipodal subset $M$ we reach in this case there exists an antipodal subset $M_{1}$ of $P_{4}(\{11, \ldots, n\})$ which satisfies $M=A_{4,3} \cup M_{1}$. However, this does not happen, because $A_{4,3}$ is not a maximal antipodal subset of $P_{4}(10)$.

(2) We take the minimum element $\{5,6,7,8\}$ of the orbit $P_{4}(\{5, \ldots, n\})$, add it to $A_{1}$ and obtain

$$
A_{2,2}=\{\{1,2,3,4\},\{5,6,7,8\}\} .
$$

We have

$$
\begin{aligned}
A\left(A_{2,2}\right)= & P_{2}(\{1,2,3,4\}) \times P_{2}(\{5,6,7,8\}) \\
& \cup P_{2}(\{1,2,3,4\}) \times P_{2}(\{9, \ldots, n\}) \\
& \cup P_{2}(\{5,6,7,8\}) \times P_{2}(\{9, \ldots, n\}) \\
& \cup P_{4}(\{9, \ldots, n\}) .
\end{aligned}
$$

The stabilizer $S\left(A_{2,2}\right)$ induces permutations on $\{1,2,3,4\}$ and $\{5,6,7,8\}$ and interchanging these two, so it acts transitively on each of

$$
\begin{aligned}
& P_{2}(\{1,2,3,4\}) \times P_{2}(\{5,6,7,8\}), \\
& P_{2}(\{1,2,3,4\}) \times P_{2}(\{9, \ldots, n\}) \cup P_{2}(\{5,6,7,8\}) \times P_{2}(\{9, \ldots, n\}), \\
& P_{4}(\{9, \ldots, n\}) .
\end{aligned}
$$

These are three orbits of $S\left(A_{2,2}\right)$. Hence we divide the procedure to three cases.

(2.1) We take the minimum element $\{1,2,5,6\}$ of the orbit $P_{2}(\{1,2,3,4\}) \times$ $P_{2}(\{5,6,7,8\})$ and add it to $A_{2,2}$. This reduces to the case (1).

(2.2) We take the minimum element $\{1,2,9,10\}$ of the orbit

$$
P_{2}(\{1,2,3,4\}) \times P_{2}(\{9, \ldots, n\}) \cup P_{2}(\{5,6,7,8\}) \times P_{2}(\{9, \ldots, n\})
$$

and add it to $A_{2,2}$. This reduces to the case (1).

(2.3) We take the minimum element $\{9,10,11,12\}$ of the orbit $P_{4}(\{9, \ldots, n\})$ and add it to $A_{2,2}$. When we proceed the procedure in this case, if we take one element of the orbits of (2.1) and (2.2), this reduces to the cases already mentioned above. Thus it is sufficient to take elements of $P_{4}(\{9, \ldots, n\})$. For any maximal antipodal subset $M$ we reach in this case there exists an antipodal subset $M_{1}$ of $P_{4}(\{9, \ldots, n\})$ which 
satisfies $M=A_{2,2} \cup M_{1}$. However, this does not happen, because $A_{2,2}$ is not a maximal antipodal subset of $P_{4}(8)$.

Example 6.1. We show the maximal antipodal subsets of $P_{4}(11)$.

$$
A(4,10), \quad B(4,8), \quad B(4,7) \cup(A(4,4)+7) .
$$

Corollary 6.1. Any disjoint union of p subsets congruent with $A(4,2 l)$ where $l \geq 2$ and $l \neq 4$ hold, $q$ subsets congruent with $B(4,7)$ and $r$ subsets congruent with $B(4,8)$ is a maximal antipodal subset of $P_{4}(n)$ in the following cases:

(1) $p=0,1, q>0, r \geq 0, n=2 l p+7 q+8 r$,

(2) $p=1, q=0, r \geq 0, n=2 l p+7 q+8 r, 2 l p+7 q+8 r+1$,

(3) $p=0, q=0, r>0, n=2 l p+7 q+8 r, 2 l p+7 q+8 r+1,2 l p+$ $7 q+8 r+2,2 l p+7 q+8 r+3$.

Conversely any maximal antipodal subset of $P_{4}(n)$ is congruent with one of the above subsets.

Proof. By Theorem 6.1 any maximal antipodal subset of $P_{4}(n)$ is equal to a disjoint union of $p$ subsets congruent with $A(4,2 l)$ where $l \geq 2$ and $l \neq 4$ hold, $q$ subsets congruent with $B(4,7)$ and $r$ subsets congruent with $B(4,8)$. We have to find a necessary and sufficient condition that such a disjoint union is a maximal antipodal subset of $P_{4}(n)$. Since $A(4,2 l) \cup(A(4,2 m)+2 l) \subset A(4,2(l+m))$, we have $p=0,1$. We consider two cases where $q>0$ and $q=0$. Because of the relation $B(4,7) \subset B(4,8)$, we see that $B(4,7)$ is not a maximal antipodal subset of $P_{4}(8)$. In the case where $q>0$ we have $n=2 l p+7 q+8 r$. We suppose $q=0$. If $l$ is not four, $A(4,2 l)$ is a maximal antipodal subset of $P_{4}(2 l)$ and $P_{4}(2 l+1)$ but is not maximal in $P_{4}(2 l+2) . B(4,8)$ is a maximal antipodal subset of $P_{4}(8), P_{4}(9), P_{4}(10)$ and $P_{4}(11)$ but is not maximal in $P_{4}(12)$. Therefore in the case where $p=1$ we have $n=2 l p+7 q+8 r, 2 l p+7 q+8 r+1$ and in the case where $p=0$ we have $n=2 l p+7 q+8 r, 2 l p+7 q+8 r+1,2 l p+7 q+8 r+2,2 l p+7 q+8 r+3$.

Corollary 6.2. The 2-number $\#_{2} \tilde{G}_{4}\left(\mathbb{R}^{n}\right)$ is as follows:

\begin{tabular}{|c|c|c|c|c|c|}
\hline$n$ & 5 & 6 & 7 & $8, \ldots, 11$ & more than 11 \\
\hline$\#_{2} \tilde{G}_{4}\left(\mathbb{R}^{n}\right)$ & 2 & 6 & 14 & 28 & {$[n / 2]([n / 2]-1)$} \\
\hline
\end{tabular}

Proof. We have

$$
\# A(4,2 l)=\left(\begin{array}{l}
l \\
2
\end{array}\right)=\frac{1}{2} l(l-1), \quad \# B(4,7)=7, \quad \# B(4,8)=14
$$

and obtain the corollary by Corollary 3.1. 
Remark 6.1. Some 4-forms correspond to maximal antipodal subsets of $P_{4}(n)$ in a way similar to Remark 4.2. The half of the second power product of the Kähler form on $\mathbb{C}^{l}=\mathbb{R}^{2 l}$ corresponds to $A(4,2 l)$. The fundamental 4-form on $\mathbb{H}^{2}$ defined by Kraines [3] corresponds to $B(4,8)$. This form is invariant under the action of $S p(2) S p(1)$.

\section{Maximal antipodal SUbSets of $P_{k}(n)$ FOR Higher $k$}

We show some results on maximal antipodal subsets of $P_{k}(n)$ for higher $k$, by generalizing some arguments in previous sections.

For $k=2 k^{\prime}$ we define

$$
A(k, 2 l)=\left\{\alpha_{1} \cup \cdots \cup \alpha_{k^{\prime}} \in P_{k}(2 l) \mid \alpha_{i} \in\{\{1,2\}, \ldots,\{2 l-1,2 l\}\}\right\} .
$$

We can see easily that $A(k, 2 l)$ is an antipodal subset of $P_{k}(2 l)$. This is a generalization of $A(4,2 l)$ defined in the previous section.

Proposition 7.1. Let $k=2 k^{\prime}$. If $l \geq 3 k^{\prime}-1$, then $A(k, 2 l)$ is a maximal antipodal subset of $P_{k}(2 l)$ and $P_{k}(2 l+1)$.

Proof. We suppose that $A(k, 2 l)$ is not a maximal antipodal subset of $P_{k}(2 l)$. We can take $\beta \in P_{k}(2 l)-A(k, 2 l)$ which is antipodal with every elements of $A(k, 2 l)$. Since $\beta \notin A(k, 2 l)$, there exists $1 \leq i \leq l$ satisfying $\#(\beta \cap\{2 i-1,2 i\})=1$. Let

$$
B=\{\{1,2\}, \ldots,\{2 l-1,2 l\}\} .
$$

Since $\# \beta=k$, there exist $\alpha_{1}, \ldots, \alpha_{k} \in B$ satisfying $\beta \subset \alpha_{1} \cup \cdots \cup \alpha_{k}$. $B-\left\{\alpha_{1}, \ldots, \alpha_{k}\right\}$ has $l-k$ elements and $l-k \geq 3 k^{\prime}-1-2 k^{\prime}=k^{\prime}-1$. Hence we can take

$$
\begin{gathered}
\beta_{1}, \ldots, \beta_{k^{\prime}-1} \in B-\left\{\alpha_{1}, \ldots, \alpha_{k}\right\} . \\
\{2 i-1,2 i\} \cup \beta_{1} \cup \cdots \cup \beta_{k^{\prime}-1} \in A(k, 2 l) \text { and the cardinality of } \\
\beta \cap\left(\{2 i-1,2 i\} \cup \beta_{1} \cup \cdots \cup \beta_{k^{\prime}-1}\right)=\beta \cap\{2 i-1,2 i\}
\end{gathered}
$$

is one, thus $\beta$ and $\{2 i-1,2 i\} \cup \beta_{1} \cup \cdots \cup \beta_{k^{\prime}-1}$ are not antipodal, which is a contradiction. Therefore $A(k, 2 l)$ is a maximal antipodal subset of $P_{k}(2 l)$.

Next we suppose that $A(k, 2 l)$ is not a maximal antipodal subset of $P_{k}(2 l+1)$. We can take take $\beta \in P_{k}(2 l+1)-A(k, 2 l)$ which is antipodal with every elements of $A(k, 2 l)$. Since $A(k, 2 l)$ is a maximal antipodal subset of $P_{k}(2 l), \beta$ contains $2 l+1$. The cardinality of $\# \beta$ is even, thus there is $1 \leq i \leq l$ satisfying $\#(\beta \cap\{2 i-1,2 i\})=1$. Hence we can see that this is a contradiction in a way similar to the previous case. Therefore $A(k, 2 l)$ is a maximal antipodal subset of $P_{k}(2 l+1)$. 
Remark 7.1. The $1 / k^{\prime}$ ! times the $k^{\prime}$-th power product of the Kähler form on $\mathbb{C}^{l}=\mathbb{R}^{2 l}$ corresponds to $A\left(2 k^{\prime}, 2 l\right)$.

In order to construct a maximal antipodal subset including $A(4 m, 8 m)$ in $P_{4 m}(8 m)$, we prepare the following lemma.

Lemma 7.1. For $\alpha=\left\{\alpha_{1}, \ldots, \alpha_{2 m}\right\} \in P_{1}(\{1,2\}) \times \cdots \times P_{1}(\{4 m-$ $1,4 m\}) \subset P_{2 m}(4 m)$ we define

$$
\alpha^{e}=\left\{i \mid \alpha_{i} \text { is even }\right\}, \quad \alpha^{o}=\left\{i \mid \alpha_{i} \text { is odd }\right\} .
$$

For any $\alpha, \beta \in P_{1}(\{1,2\}) \times \cdots \times P_{1}(\{4 m-1,4 m\})$ we have

$$
\#(\alpha \cap \beta)=2 \#\left(\alpha^{e} \cap \beta^{e}\right)+\# \beta^{o}-\# \alpha^{e} .
$$

Proof. We note that

$$
\alpha^{e} \cup \alpha^{o}=\beta^{e} \cup \beta^{o}=\{1, \ldots, 2 m\}
$$

are disjoint unions. We have

$$
\alpha \cap \beta=\left\{\alpha_{i} \mid i \in \alpha^{e} \cap \beta^{e}\right\} \cup\left\{\alpha_{i} \mid i \in \alpha^{o} \cap \beta^{o}\right\},
$$

which is a disjoint union. Thus we obtain

$$
\begin{aligned}
\#(\alpha \cap \beta) & =\#\left(\alpha^{e} \cap \beta^{e}\right)+\#\left(\alpha^{o} \cap \beta^{o}\right) \\
& =\#\left(\alpha^{e} \cap \beta^{e}\right)+\left(\# \beta^{o}-\#\left(\alpha^{e} \cap \beta^{o}\right)\right) \\
& =2 \#\left(\alpha^{e} \cap \beta^{e}\right)+\# \beta^{o}-\# \alpha^{e} .
\end{aligned}
$$

We define $E v_{4 m}$ for a natural number $m$ by

$$
\begin{gathered}
E v_{4 m}=\left\{\left\{\alpha_{1}, \ldots, \alpha_{2 m}\right\} \mid \alpha_{i} \in\{2 i-1,2 i\}(1 \leq i \leq 2 m),\right. \\
\text { the number of even numbers is even }\} .
\end{gathered}
$$

This is a subset of $P_{1}(\{1,2\}) \times P_{1}(\{3,4\}) \times \cdots \times P_{1}(\{4 m-1,4 m\}) \subset$ $P_{2 m}(4 m)$ and a generalization of $E v_{8}$ defined in the proof of Theorem 6.1 .

Proposition 7.2. $A(4 m, 8 m) \cup E v_{8 m}$ is a maximal antipodal subset of $P_{4 m}(8 m)$ for $m \geq 1$.

Proof. We first show that $A(4 m, 8 m) \cup E v_{8 m}$ is an antipodal subset of $P_{4 m}(8 m)$. For any $\alpha$ in $A(4 m, 8 m)$ and $\beta$ in $E v_{8 m}, \#(\alpha \cap \beta)=2 m$ by their definitions, thus they are antipodal. We take any elements $\alpha$ and $\beta$ in $E v_{8 m}$. Lemma 7.1 implies

$$
\#(\alpha \cap \beta)=2 \#\left(\alpha^{e} \cap \beta^{e}\right)+\# \beta^{o}-\# \alpha^{e},
$$

which is even. Hence $\#(\alpha \cap \beta)$ is even and $\alpha$ and $\beta$ are antipodal. Therefore $A(4 m, 8 m) \cup E v_{8 m}$ is antipodal. 
Next we show that $A(4 m, 8 m) \cup E v_{8 m}$ is a maximal antipodal subset of $P_{4 m}(8 m)$. For this purpose we show that for any $\alpha$ in $P_{4 m}(8 m)-$ $A(4 m, 8 m) \cup E v_{8 m}$ there exists an element in $A(4 m, 8 m) \cup E v_{8 m}$ which is not antipodal to $\alpha$. There are two posibilities of $\alpha$ as follows:

(1) $\#(\alpha \cap\{2 i-1,2 i\})=2$ for some $i$ and $\#(\alpha \cap\{2 j-1,2 j\})=1$ for some $j$,

(2) $\alpha \in P_{1}(\{1,2\}) \times \cdots \times P_{1}(\{8 m-1,8 m\})-E v_{8 m}$.

In the case $(1)$, we put

$$
\begin{aligned}
& \{i \mid \#(\alpha \cap\{2 i-1,2 i\})=2\}=\left\{i_{1}, \ldots, i_{a}\right\}, \\
& \{j \mid \#(\alpha \cap\{2 j-1,2 j\})=1\}=\left\{j_{1}, \ldots, j_{b}\right\} .
\end{aligned}
$$

These imply $4 m=\# \alpha=2 a+b$, hence $b$ is even. Thus $2 m=a+b / 2$. In the case where $b / 2$ is odd, we put

$$
\begin{aligned}
& \beta=\left\{2 i_{1}-1,2 i_{1}\right\} \cup \cdots \cup\left\{2 i_{a}-1,2 i_{a}\right\} \\
& \cup\left\{2 j_{1}-1,2 j_{1}\right\} \cup \cdots \cup\left\{2 j_{b / 2}-1,2 j_{b / 2}\right\} \in A(4 m, 8 m)
\end{aligned}
$$

and

$$
\#(\alpha \cap \beta)=2 a+\frac{b}{2}
$$

is odd, hence $\alpha$ and $\beta$ are not antipodal. In the case where $b / 2$ is even, we take $i_{*}$ satisfying

$$
\alpha \cap\left\{2 i_{*}-1,2 i_{*}\right\}=\emptyset
$$

We put

$$
\begin{aligned}
& \gamma=\left\{2 i_{1}-1,2 i_{1}\right\} \cup \cdots \cup\left\{2 i_{a}-1,2 i_{a}\right\} \\
& \quad \cup\left\{2 j_{1}-1,2 j_{1}\right\} \cup \cdots \cup\left\{2 j_{b / 2-1}-1,2 j_{b / 2-1}\right\} \cup\left\{2 i_{*}-1,2 i_{*}\right\} \in A(4 m, 8 m)
\end{aligned}
$$

and

$$
\#(\alpha \cap \gamma)=2 a+\frac{b}{2}-1
$$

is odd, hence $\alpha$ and $\gamma$ are not antipodal.

In the case (2), $\# \alpha^{e}$ is odd. For any $\delta \in E v_{8 m}$ we obtain

$$
\#(\alpha \cap \delta)=2 \#\left(\alpha^{e} \cap \delta^{e}\right)+\# \delta^{o}-\# \alpha^{e}
$$

by Lemma 7.1 . Hence $\#(\alpha \cap \delta)$ is odd, and $\alpha, \delta$ are not antipodal.

Anyway there exists an element in $A(4 m, 8 m) \cup E v_{8 m}$ which is not antipodal to $\alpha$. Therefore $A(4 m, 8 m) \cup E v_{8 m}$ is a maximal antipodal subset in $P_{4 m}(8 m)$.

Remark 7.2. We have already proved the statement of Proposition 7.2 in the case $m=1$ in the proof of Theorem 6.1 , where we showed $A(4,8) \cup E v_{8}=A_{14}$ is congruent with $B(4,8)$ in $P_{4}(8)$. Proposition 7.1 shows that $A(k, 2 l)$ is a maximal antipodal subset of $P_{k}(2 l)$ and 
$P_{k}(2 l+1)$ for $l \geq 3 k^{\prime}-1$. On the other hand, $A(4 m, 8 m)$ is not a maximal antipodal subset of $P_{4 m}(8 m)$ by Proposition 7.2. Moreover the case of $m=1$ shows that the condition $l \geq 3 k^{\prime}-1$ of Proposition 7.1 is sharp.

Lemma 7.2. If $A \subset P_{k}(n)$ is an antipodal subset, then $A \times\{\{n+1\}\}=$ $\{\sigma \cup\{n+1\} \mid \sigma \in A\}$ is an antipodal subset of $P_{k+1}(n+1)$.

Proof. For $\alpha, \beta \in A$ we have

$$
(\beta \cup\{n+1\})-(\alpha \cup\{n+1\})=\beta-\alpha .
$$

Thus $A \times\{\{n+1\}\}$ is antipodal, if $A$ is antipodal.

For a subset $A \subset P_{k}(2 k+1)$, we have $A^{c} \subset P_{k+1}(2 k+1)$ and $A \times\{\{2 k+2\}\} \subset P_{k+1}(2 k+2)$. Using these we define the twisted double $T D(A)$ of $A$ by

$$
T D(A)=A^{c} \cup A \times\{\{2 k+2\}\} .
$$

This is a generalization of the construction of $B(4,8)$ from $B(3,7)$ in the previous section. In the case where $k=3$, we have $T D(B(3,7))=$ $B(4,8)$.

Proposition 7.3. We assume that $k$ is odd. If $A \subset P_{k}(2 k+1)$ is an antipodal subset, then $T D(A)$ is an antipodal subset of $P_{k+1}(2 k+2)$. Moreover, if $A$ is a maximal antipodal subset of $P_{k}(2 k+1)$, then $T D(A)$ is a maximal antipodal subset of $P_{k+1}(2 k+2)$.

Proof. If $A$ is antipodal, $A^{c}$ is an antipodal subset of $P_{k+1}(2 k+1)$ by Lemma 4.1, and $A \times\{\{2 k+2\}\}$ is an antipodal subset of $P_{k+1}(2 k+2)$ by Lemma 7.2. In order to prove that $T D(A)$ is antipodal, it is sufficient to show that $\alpha^{c}$ and $\beta \cup\{2 k+2\}$ are antipodal for $\alpha, \beta \in A$. We have

$$
\text { (*) } \quad \alpha^{c} \cap(\beta \cup\{2 k+2\})=\beta-\alpha,
$$

where $\alpha^{c}$ is the complement of $\alpha$ in $\{1, \ldots, 2 k+1\}$. The cardinality of $(*)$ is even and $k+1$ is even, thus $\alpha^{c}$ and $\beta \cup\{2 k+2\}$ are antipodal in $P_{k+1}(2 k+2)$.

We suppose that $A$ is a maximal antipodal subset of $P_{k}(2 k+1)$. We take an element $\gamma \in P_{k+1}(2 k+2)$ which is antipodal with every elements of $T D(A)$. In the case where $\gamma$ does not contain $2 k+2, \gamma$ belongs to $P_{k+1}(2 k+1) . A^{c}$ is a maximal antipodal subset of $P_{k+1}(2 k+1)$ and $\gamma$ is antipodal with every elements of $A^{c}$, hence $\gamma \in A^{c} \subset T D(A)$. In the case where $\gamma$ contains $2 k+2$, there is $\delta$ in $P_{k}(2 k+1)$ satisfying $\gamma=\delta \cup\{2 k+2\}$. For any $\beta \in A \gamma$ and $\beta \cup\{2 k+2\}$ are antipodal, thus the cardinality of

$$
(\beta \cup\{2 k+2\})-(\delta \cup\{2 k+2\})=\beta-\delta
$$


is even. Hence $\beta$ and $\delta$ are antipodal. Because of the maximality of $A$ in $P_{k}(2 k+1)$ we get $\delta \in A$ and $\gamma \in A \times\{\{2 k+2\}\} \subset T D(A)$. In any case we have $\gamma \in T D(A)$. Therefore $T D(A)$ is a maximal antipodal subset of $P_{k+1}(2 k+2)$.

\section{ACKNOWLEDGMENTS}

The author would like to thank Makiko Tanaka for useful discussion on the subject of this paper during preparing the manuscript. He is also indebted to the referee, whose comments improved the manuscript.

The author was partly supported by the Grant-in-Aid for Science Research (C) 2012 (No. 24540064), Japan Society for the Promotion of Science.

\section{REFERENCES}

[1] B.-Y. Chen and T. Nagano, A Riemannian geometric invariant and its applications to a problem of Borel and Serre, Trans. Amer. Math. Soc. 308 (1988), 273-297.

[2] R. Harvey and B.H. Lawson, ,Jr., Calibrated geometry. Acta Math. 148 (1982), $47-157$.

[3] V.Y. Kraines, Topology of quaternionic manifolds. Trans. Amer. Math. Soc. 122 (1966), 357 - 367.

[4] M. S. Tanaka and H. Tasaki, Antipodal sets of symmetric $R$-spaces, Osaka J. Math. 50 no.1 (2013), 161-169.

Division of Mathematics,, Faculty of Pure and Applied Sciences, University of TsukubA,, TsukubA, IBARAKI, 305-8571 JAPAN

E-mail address: tasaki@math.tsukuba.ac.jp 